\title{
Crosstalk between TEMs and endothelial cells modulates angiogenesis and metastasis via IGF1-IGF1R signalling in epithelial ovarian
} cancer

Xinjing Wang ${ }^{1,7}$, Qinyi Zhu ${ }^{1,7}$, Yingying $\mathrm{Lin}^{2,7}$, Li Wu ${ }^{3}$, Xiaoli Wu ${ }^{1}$, Kai Wang ${ }^{4}$, Qizhi He ${ }^{5}$, Congjian $\mathrm{Xu}^{6}$, Xiaoping Wan $^{3}$ and Xipeng Wang ${ }^{\star, 1}$

${ }^{1}$ Department of Gynecology and Obstetrics, XinHua Hospital, Shanghai JiaoTong University School of Medicine, Shanghai 200092, China; ${ }^{2}$ Department of Neurosurgery, Ren Ji Hospital, School of Medicine, Shanghai JiaoTong University, Shanghai 200127, China; ${ }^{3}$ Department of Gynecology, Shanghai First Maternity and Infant Hospital, Tongji University School of Medicine, Shanghai 200126, China; ${ }^{4}$ Central Laboratory, Shanghai First Maternity and Infant Hospital, Tongji University School of Medicine, Shanghai 200126, China; ${ }^{5}$ Department of Pathology, Shanghai First Maternity and Infant Hospital, Tongji University School of Medicine, Shanghai 200126, China and 'Department of Gynecology, Obstetrics and Gynecology Hospital of Fudan University, Shanghai 200011, China

Background: Epithelial ovarian cancer (EOC) is the leading cause of death from gynaecologic malignancies and has a poor prognosis due to metastasis. Drugs targeting the angiogenesis pathway significantly improve patient outcome. However, the key factors linking angiogenesis and metastasis have not been elucidated. In this study, we found Tie2 expressing monocytes $\left(\mathrm{CD} 14^{+} \mathrm{Tie}^{+}, \mathrm{TEMs}\right)$ as key contributors to angiogenesis and metastasis of EOC.

Methods: Tissue slides were evaluated by immunofluorescence for the presence of total tissue macrophages and TEMs. The correlation between microvascular density (MVD) values and the TEMs number or ratio was calculated in both ovarian cancer tissues and peritoneum. The rate of TEMs in monocytes was evaluated in the peripheral blood of female healthy donors, benign cysts patients, and EOC patients using flow cytometry. The TEMs rate in ascites from EOC patients was also evaluated by flow cytometry. The concentration of Ang2, as the ligand of Tie2, was examined by ELISA in serum samples of EOC patients, benign cysts patients, and ascites samples of EOC patients. The effects of Ang2 on the migration and the cytokine expression of TEMs were further examined. The pro- angiogenesis activity of TEMs via IGF1 was performed in both in vivo and in vitro. And the IGF1 blocking test was performed using neutralising antibody.

Results: TEMs were significantly higher in tumour foci, peripheral blood and ascites in EOC patients. The proportion of TEMs among total tissue macrophages was positively correlated with tumour MVD. In vivo animal results showed that TEMs promoted EOC angiogenesis and metastasis. Further functional and mechanisms studies revealed that concentration of angiopoietin 2 (Ang2), a ligand of Tie2, was elevated in EOC ascites which further recruit TEMs in a dose-dependent manner as a powerful chemokine to TEMs. Recruited TEMs promoted endothelial cell function through IGF1-activated downstream signalling. Blocking secreted IGF1 using inhibiting antibody reduced TEMs mediated angiogenesis and metastasis.

Conclusions: TEMs significantly increased in EOC patients and were recruited to tumour loci by the increased Ang2. The increased TEMs have diagnostic value in ovarian cancer and were positively correlated with the MVD in ovarian cancer tissue. Furthermore, TEMs promote angiogenesis via IGF1 in both in vivo and in vitro experimental systems after stimulation by Ang2. Altogether, this study paves the way to develop novel therapy targets as the axis of Ang2-TEMs-IGF1 in EOC.

${ }^{\star}$ Correspondence: Professor X Wang; E-mail: wangxipeng@xinhuamed.com.cn

Received 29 June 2017; revised 3 August 2017; accepted 4 August 2017;

published online 12 September 2017

C The Author(s) named above 
Epithelial ovarian cancer (EOC) is the most deadly cancer of the female genital tract and ranks as the seventh leading cause of all cancer-related deaths among women worldwide (Jemal et al, 2011). Nearly $70 \%$ of EOC patients are diagnosed at an advanced stage, presenting with widely distributed metastases on the surface of peritoneum, and displaying a poor prognosis caused by the progression of the disease (Seidman et al, 2004).

The peritoneum is organised to protect the integrity of intraabdominal organs, which facilitates infiltration of inflammatory cells to sites of tumour implants and might also serve to facilitate the promotion of tumour growth and spread. Our previous study has suggested that more than $75 \%$ of immune cells infiltrated in the peritoneum of EOC were $\mathrm{CD}^{+} 8^{+}$macrophage with $\mathrm{M} 2$ polarisation, and less than $25 \%$ were $\mathrm{CD}^{+} \mathrm{T}$ cells (Wang et al, 2006). Examination of surgically resected EOC samples revealed frequent changes in the peritoneum harbouring tumour, including thickening or oedema, enhanced vascular patterns, and soft or firm adhesions. The peritoneum and intestinal serosa sometimes exhibited a florid appearance, similar to that found in peritonitis. Significant infiltration by macrophage was one of the feature of chronic inflammation. These tumour-associated macrophage could promote angiogenesis by interaction with EOC cells (Wang et al, 2013).

Recently, a novel subset of monocytes that expressed Tie2 (also known as the receptor tyrosine kinase, TEK) has been identified as Tie2 expressing monocytes (TEMs) in mouse models, human peripheral blood and several human tumour tissues (De Palma et al, 2003; De Palma et al, 2005; Venneri et al, 2007; Pucci et al, 2009). Increased numbers of TEMs in peripheral blood and liver are under consideration as novel diagnostic markers for hepatocellular carcinoma (De Palma et al, 2013; Matsubara et al, 2013). Tie2, the receptor for angiopoietins (especially angiopoietin 2, Ang2), has important functions in angiogenesis induced by the Ang2-Tie2 axis (Saharinen et al, 2008). Endothelial cells were previously believed to be the only cells that express Tie2 (Partanen et al, 1992). The specific pro-angiogenic role of TEMs has been confirmed in many studies of human tumours and non-neoplastic diseases, and this novel subset of monocytes is considered as a strong angiogenesis-promoting entity, perhaps more potent than canonical tumour-associated macrophages (Ribatti, 2009; Coffelt et al, 2010; Huang et al, 2011). Although the potency TEMs for promoting angiogenesis and their reaction to Ang2 stimulation has been reported in several human cancers, their presence, frequency and their roles in the angiogenesis promotion of EOC remain unclear.

In this study, we examined tumours from patients with EOC and benign cysts for the incidence of TEMs and their infiltration characteristics, and correlated these features with microvascular density (MVD). We evaluated Tie2 over-expressing monocytes in angiogenesis assays in vitro and in vivo to further explore the mechanism of angiogenesis-promoting activities by these cells. Elevated IGF1 secretion by TEMs was found after Ang2 stimulation. Activation of the downstream IGF1 pathway was verified in endothelial cells, as evidenced by phosphorylation of the IGF1R, together with the phosphorylation of its downstream molecules Erk1/2 and Akt.

Taken together, results of this study suggest that the axis of Ang2-TEMs-IGF1 could enhance angiogenesis by activation of the IGFR pathway in endothelial cells during progression of EOC. Targeted therapy that affects this axis in EOC might be warranted in the future.

\section{MATERIALS AND METHODS}

Patients and tissue samples. Our study was approved by the Ethics Committee of the Shanghai First Maternity and Infant Hospital, School of Medicine, Tongji University. A total of 124 patients with histologically confirmed EOC at Shanghai First Maternity and Infant Hospital, Tongji University between January 2012 and December 2014 were recruited for this study. Their diagnoses were independently reviewed by two pathologists, classified by using WHO criteria. All the patients were informed with the informed consents obtained in the present study.

Cell lines. Human monocytic cell line U937 and human ovarian cancer cell line SKOV3 were obtained from ATCC in December 2013 and the cell lines were authenticated by STR test. The last time that the cell lines were tested was December 2015.

Immunofluorescence and immunohistochemistry. $\mathrm{CD}^{+} 8^{+}$ Tie ${ }^{+}$TEMs and $\mathrm{CD}^{+} 8^{+} \mathrm{Tie}^{-}$macrophages in patient tissue slides were detected using mouse anti-human Tie2 (clone Ab33, Millipore, Billerica, MA, USA) and rabbit anti-human CD68 (MAB20401, R\&D Systems, Minneapolis, MN, USA) antibodies, followed by Alexa Fluor 488-conjugated goat anti-mouse IgG (104546, Jackson, Lancaster, PA, USA) and Cy3-conjugated goat anti-rat IgG (99002, Jackson) as secondary antibodies. All cell nuclei were counterstained with DAPI (D9542, Sigma, St Louis, MO, USA).

Immunohistochemical analysis of cluster of differentiation 31 (CD31) was performed with rabbit anti-CD31 antibody (ab28364, Abcam, Cambridge, UK). IGF1 expression was evaluated by immunohistochemical staining using the anti-IGF1 antibody (ab9572, Abcam). HPR-conjugated goat anti-rabbit antibody (31461, Invitrogen, Carlsbad, CA, USA) was used as the secondary antibody. Images were captured by optical microscope, and MVD and an IGF1 immunostaining score were calculated from five random high power fields at $\times 20$ magnifications. For IGF1 immunostaining scoring, the quantitative scoring method was used as follows: the intensity of staining was firstly divided into weak staining (score $=1)$, moderate staining $($ score $=2)$ and strong staining (score $=3$ ). Then the positive cells ratio was measured as $<10 \%($ score $=0), 10-25 \%($ score $=1), 25-50 \%$ (score $=2), 50$ $75 \%($ score $=3),>75 \%$ (score $=4)$. We multiply the intensity of staining score by the positive cells ratio score to get the final immunostaining score of IGF1. The immunohistochemical results were valued manually by two different pathologists.

Detection of TEMs prevalence in peripheral blood and ascites. Peripheral blood mononuclear cells (PBMCs) and cells in ascites isolated from patient cohorts were double-stained with FITCconjugated anti-CD14 (555397, BD Biosciences, Franklin, NJ, USA) and APC-conjugated anti-Tie2 (FAB3131A, R\&D Systems) antibodies. Tie $2^{+} \mathrm{CD} 14^{+}$TEMs were detected by flow cytometry. Numbers of TEMs were quantified by gating on $\mathrm{CD}_{1} 4^{+}$cells. FITC/APC-conjugated isotype antibodies were used control antibodies.

TEMs isolation and culture. Monocytes were enriched from PBMCs by positive immunomagnetic selection using anti-CD14 MicroBeads (130-050-201, Miltenyi, Cologne, Germany). Tie2 ${ }^{+}$ $\mathrm{CD}_{14}{ }^{+}$TEMs were sorted using FITC-conjugated anti-CD14 and APC-conjugated anti-Tie2 antibodies. Sorted TEMs were cultured using RPMI 1640 medium containing 10\% FBS and 1\% penicillinstreptomycin in a humidified $37^{\circ} \mathrm{C}$ incubator with $5 \% \mathrm{CO}_{2}$.

Tie2 over-expression in U937 cells. To over-express TEK gene, the gene was amplified by PCR, with the following primers: TEKF:5'-GAGGATCCCCGGGTACCGGTCGCCACCATGGACTCTT TAGCCAGCTTAGTTCTCTG-3', TEK-R:5' -TCCTTGTAGTCCATACCGGCCGCTTCTTCAGCAGAAC AGTCAATTC- $3^{\prime}$.

Then the products of PCR were cloned into the pUbi-MCS3FLAG-SV40-EGFP Expression Vector System (Genechem, Shanghai, China). The recombinant vector pUbi-TEK was confirmed by sequencing (Shenggong, Shanghai, China). HEK293 T cells were cultured in DMEM supplemented with 10\% FBS. 
A
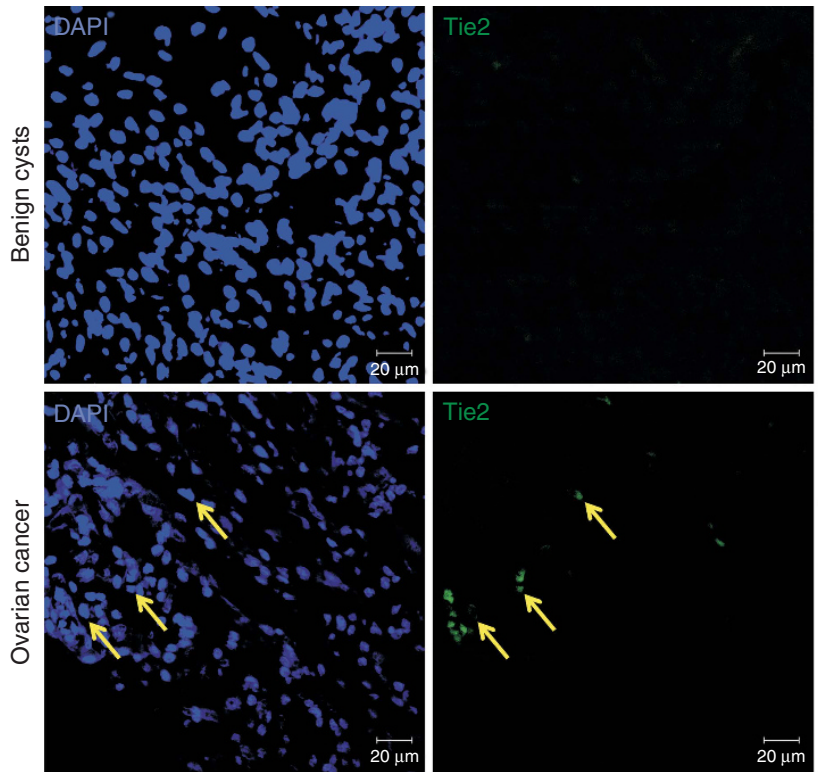
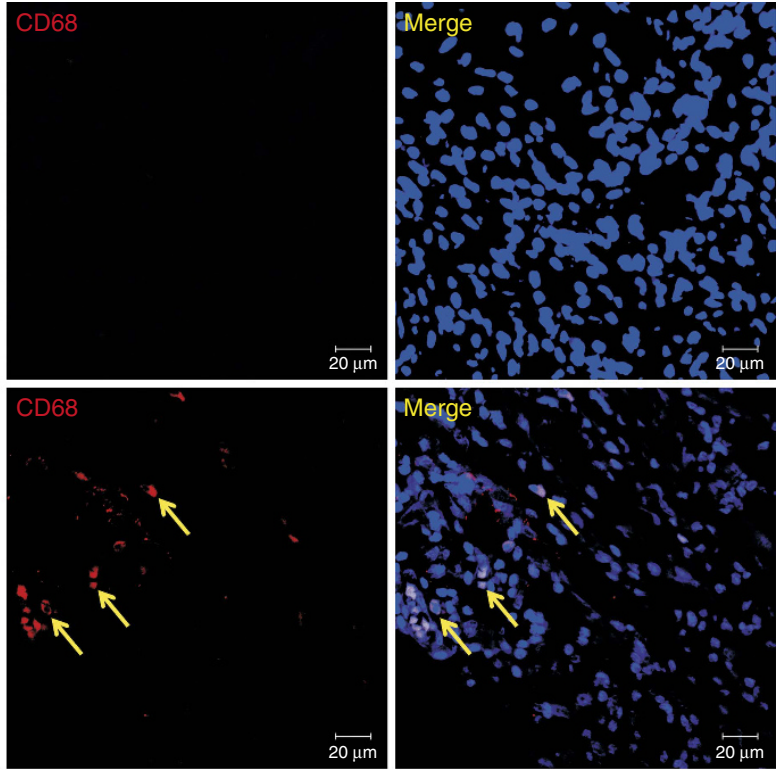

B

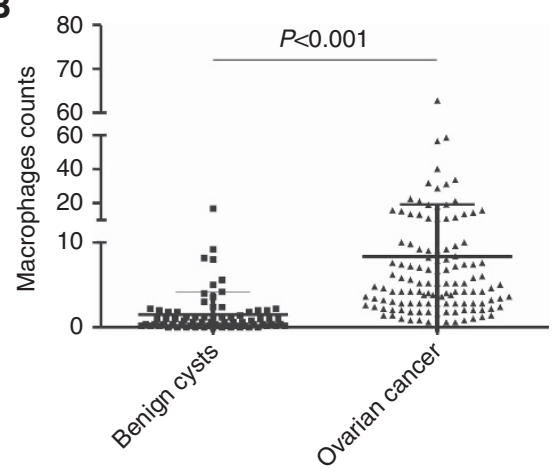

E

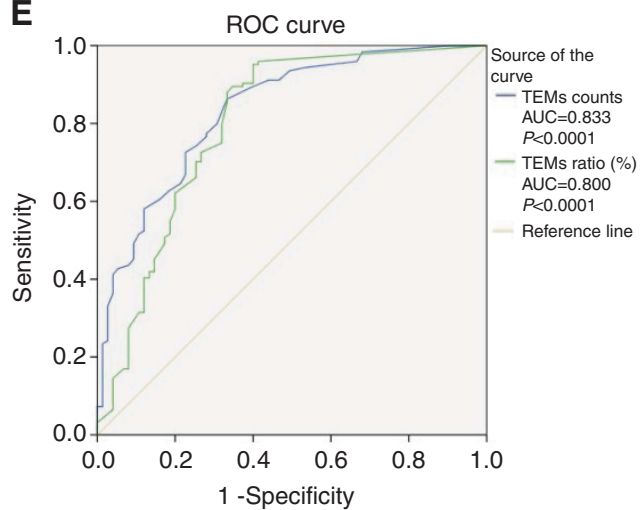

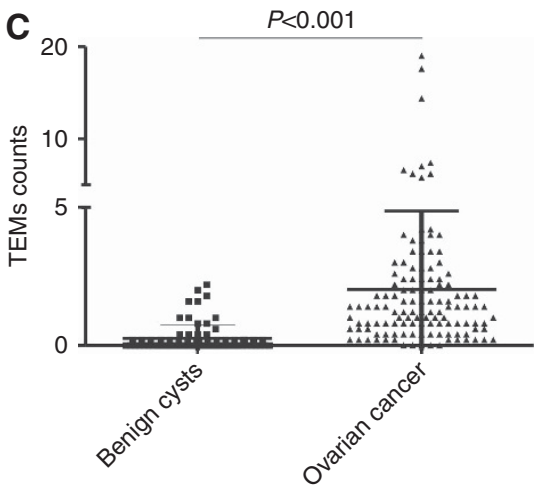

$\mathbf{F}$

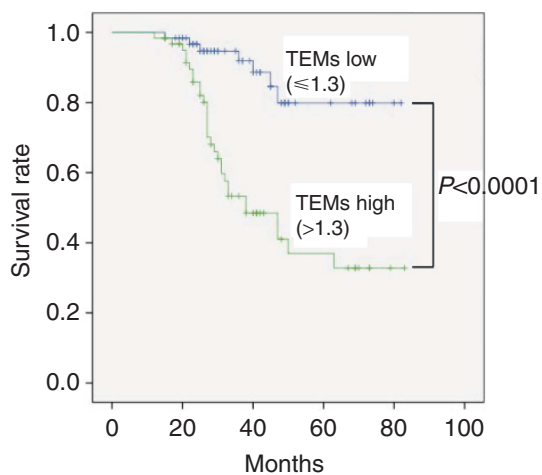

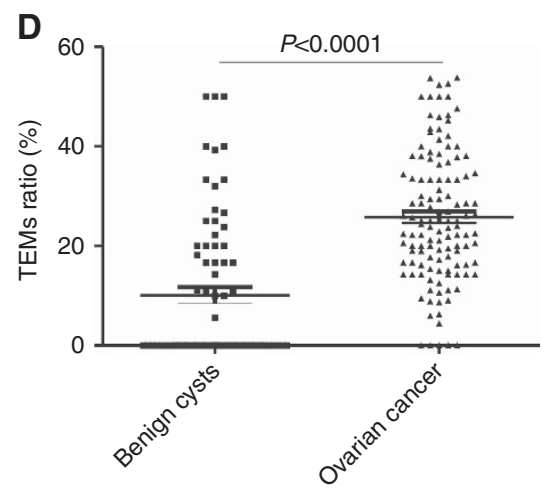

G

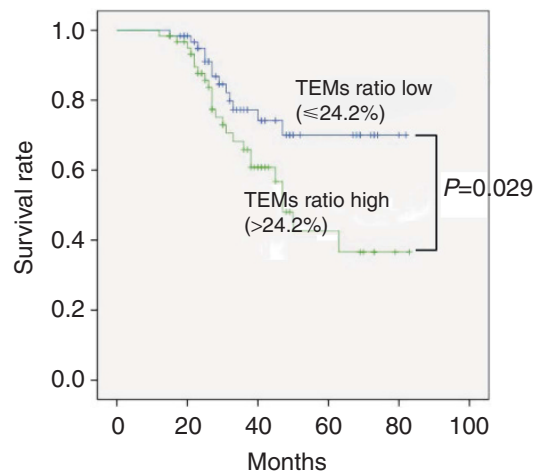

Figure 1. Increased TEMs in tissue as a biomarker for EOC and inform the patients' outcome. (A) TEMs were stained with DAPI (blue, nuclei), Tie2 (green), and CD68 (red) in benign ovarian tumour tissue $(n=75)$ and in EOC tissue $(n=124)$. (B-D) The macrophages, TEMs and the TEMs ratio were all significantly increased in EOC tissues compared with benign ovarian cysts (student's t-test). (E) ROC analyses were performed in order to assess the diagnostic value of TEM number and ratio in ovarian tissue for differentiating EOC $(n=124)$ from benign ovarian cysts $(n=75)$. $(F)$ In EOC patients, the overall survival rate was compared between patients with TEMs high (TEMs $>1.3, n=62)$ and TEM low $(T E M s \leqslant 1.3, n=62)$ using the Kaplan-Meier method, with the log-rank test for comparison. (G) In EOC patients, the overall survival rate was compared between patients with TEMs ratio high (TEMs ratio $>24.2 \%, n=62$ ) and TEMs ratio low (TEMs ratio $\leqslant 24.2 \%, n=62$ ) using the Kaplan-Meier method, with the log-rank test for comparison.

The lentivirus-mediated TEK packaging system (TEK-L.V.) was co-transfected utilising lipofectamine 2000 into HEK-293T cells in a 24-well culture plate according to the manufacturer's protocol. The supernatant was collected $48 \mathrm{~h}$ post infection, filtered with a 0.45 micrometer filter, and stored at $-80^{\circ} \mathrm{C}$. U937 cells was transduced with TEK-L.V. in the presence of $5 \mu \mathrm{g} \mathrm{ml}{ }^{-1}$ polybrene
(Sigma) and Eni.S (Genechem, Shanghai, China) for $12 \mathrm{~h}$, after which the medium was replaced by RPMI1640 supplemented with $10 \%$ FBS. The efficiency of infection of TEK-L.V. was measured using fluorescent microscope $72 \mathrm{~h}$ post infection. Moreover, the efficiency of infection is confirmed by immunofluorescence detection or FACS. For further study, $\mathrm{GFP}^{+} \mathrm{U} 937$ was isolated 
by FACS. Over-expression of TEK was determined by western blot analysis, respectively. The lentivirus-mediated non-specific control packaging system (NC-L.V.) was used as control.

TEMs and TEK-U937 migration assays. Migration assays were performed in a 24-well transwell apparatus containing 8 - $\mu \mathrm{m}$-pore inserts (BD Biosciences). Ang2 (623-AN, R\&D Systems) was diluted as the concentration of $10 \mathrm{ng} \mathrm{ml}^{-1}, 100 \mathrm{ng} \mathrm{ml}^{-1}$, or $200 \mathrm{ng} \mathrm{ml}^{-1}$ in serum-free RPMI1640 medium $(800 \mu \mathrm{l})$ at the bottom compartment of the chamber with various concentrations, and $10^{5}$ TEMs or TEK-U937 pre-treated by M-CSF (216-MC, $\mathrm{R} \& \mathrm{D}$ Systems) were resuspended in $300 \mu \mathrm{l}$ RPMI1640 then added to the top compartment. After $3 \mathrm{~h}$ incubation for TEMs or $6 \mathrm{~h}$ incubation for TEK-U937, migrating cells were labelled with $5 \mu \mathrm{g} \mathrm{ml}^{-1}$ calcein-AM (C3099, Molecular Probes, Carlsbad, CA, USA, Life Technologies, Carlsbad, CA, USA) and counted under a fluorescence microscope. For blocking analysis, TEMs were preincubated with neutralising anti-Tie 2 antibodies for $20 \mathrm{~min}$, and the Ang2 was inactivated by heat at $95^{\circ} \mathrm{C}$ for $30 \mathrm{~min}$.

Quantitative real-time PCR. The RNA of TEK-U937/NC-U937 was collected using TRIzol (15596-018, Life Technologies) before Ang2 stimulation or after Ang2 treatment for $6 \mathrm{~h}$. For q-PCR analysis, $500 \mathrm{ng}$ of total RNA was reversed transcribed to cDNA, and amplified by PCR cycling conditions: $5 \mathrm{~s}$ at $95^{\circ} \mathrm{C}$ and $30 \mathrm{~s}$ at $60{ }^{\circ} \mathrm{C}$ for 40 cycles. Differences in gene expression were determined by the $2^{\Delta \Delta} \mathrm{CT}$ method ( $\beta$-actin was used for calibration) using as: $5^{\prime}$-CCTGGCACCCAGCACAAT- $3^{\prime}$ and $5^{\prime}$-GGGCCGGACTCG TCATACT- $3^{\prime} \quad(\beta$-actin $), \quad 5^{\prime}$-CAGGATACGAACCATGAAGAT GC- $3^{\prime}$ and $5^{\prime}$-GGGG CACTGAATGGATGAAG- $3^{\prime}$ (Tie2), $5^{\prime}$-CAG GATACGAACCATGAAGATGC- $3^{\prime}$ and $5^{\prime}$-GGGGCACTGAAT GGATGAAG-3' (IL-8), 5'-CAGGATACGAACCATGAA GATG C- $3^{\prime}$ and $5^{\prime}$-GGGGCACTGAATGGATGAAG- $3^{\prime}$ (IL-10), $5^{\prime}$-CTGG AGTGTG TGCCCACTGA- $3^{\prime}$ and $5^{\prime}$-TCCTATGTGCTGGCC TTGGT-3'(VEGFA), $5^{\prime}$-TCCCACGAAATCCAGGATGC- $3^{\prime}$ and $5^{\prime}$-GGATGTTCAGGTTGACCATCAC-3' (IL-17A), 5'-GCTCTT CAGTTCGTGTGTGGA- $3^{\prime}$ and $5^{\prime}$-GCCTCCTTAGATCACAG CTCC-3'(IGF1).

Soluble protein analysis. Serum and ascites were collected from patient cohorts. The Ang2 and IGF1 levels were detected by ELISA using human Angiopoietin2 (DANG20, R\&D Systems) or human IGF1 Quantikine ELISA kit (DG100, R\&D Systems).

Table 1. Univariate and multivariate analysis of overall survival in 124 EOC patients

\begin{tabular}{|c|c|c|c|c|}
\hline \multirow[b]{3}{*}{ Variables } & \multicolumn{4}{|c|}{ Overall survival } \\
\hline & \multicolumn{2}{|c|}{$\begin{array}{c}\text { Univariate } \\
\text { analysis }\end{array}$} & \multicolumn{2}{|c|}{$\begin{array}{l}\text { Multivariate } \\
\text { analysis }\end{array}$} \\
\hline & Mean \pm s.e. & $P$ & $95 \% \mathrm{Cl}$ & $P$ \\
\hline \multicolumn{5}{|c|}{ TEMs number } \\
\hline Low ( $\leqslant 1.3)$ & $72.94 \pm 3.16$ & & & \\
\hline High $(>1.3)$ & $49.37 \pm 3.89$ & 0.001 & $1.59-8.64$ & 0.002 \\
\hline \multicolumn{5}{|c|}{ Age (years) } \\
\hline Low $(\leqslant 50)$ & $61.96 \pm 4.01$ & & & \\
\hline High $(>50)$ & $54.26 \pm 3.39$ & 0.802 & $0.45-1.67$ & 0.668 \\
\hline \multicolumn{5}{|l|}{ Grade } \\
\hline LGSC & $78.49 \pm 3.03$ & & & \\
\hline HGSC & $49.65 \pm 2.80$ & $<0.0001$ & $1.26-23.54$ & 0.023 \\
\hline \multicolumn{5}{|l|}{ MVD } \\
\hline Low $(\leqslant 4.6)$ & $65.37 \pm 3.87$ & & & \\
\hline High $(>4.6)$ & $54.47 \pm 3.92$ & 0.052 & $0.93-3.53$ & 0.083 \\
\hline \multicolumn{5}{|c|}{$\begin{array}{l}\text { Abbreviations: } \mathrm{Cl}=\text { confidence interval; } \mathrm{EOC}=\text { epithelial ovarian cancer; } \mathrm{HGSC}=\text { high } \\
\text { grade serous carcinoma; } \mathrm{LGSC}=\text { low grade serous carcinoma; } \mathrm{MVD}=\text { microvascular } \\
\text { density; s.e. = standard error; } \mathrm{TEM}=\mathrm{Tie} 2 \text { expressing monocytes. }\end{array}$} \\
\hline
\end{tabular}

EC activation assays in vitro. Conditioned medium was collected from TEMs/TEK-U937 or NC-Monocytes/NC-U937 after treatment with Ang2 $\left(100 \mathrm{ng} \mathrm{ml}^{-1}\right)$. After $6 \mathrm{~h}$ stimulation, cells were washed and the conditioned mediums were collected $24 \mathrm{~h}$ later. In the tubule formation assays, serum-starved HUVECs $\left(5 \times 10^{4}\right.$ per 96-well plate) were re-suspended in conditioned medium and seeded into growth factor-reduced Matrigel (356234, BD Biosciences). Tubules were counted after incubation for $6 \mathrm{~h}$. Recombinant human IGF-I protein (291-G1, R\&D Systems) was used as a positive control. For IGF1 block analysis, the anti-IGF1 antibody (ab9572, Abcam) was used. To determine the activation of the IGF1-IGF1R pathway in HUVECs, phosphorylation IGF1R, Akt, and Erk1/2 were detected by western blot after treatment with conditioned medium.

Mice model of ovarian cancer. Female athymic nude mice (4-6 weeks old) weighing 14-16g were purchased from Shanghai JiaoTong University School of Medicine and bred under specificpathogen-free conditions. Prior to the study, the protocols for the treatment of animals were approved by Medical Animal Care. $5 \times 10^{5}$ human ovarian cancer cell line SKOV3 transfected with luciferase (SKOV3-Luc) cells together with $1 \times 10^{5}$ TEK-U937 or NC-U937 cells in $30 \mu \mathrm{l}$ DMEM were injected into the left ovarian parenchyma of the nude mice. TEK-U937 and NC-U937 cells were stimulated by Ang2 $\left(100 \mathrm{ng} \mathrm{ml}^{-1}\right)$ for $24 \mathrm{~h}$ before injection. Then all the nude mice were separated into three groups according to the injected cell types as follows: TEK-U937 group (SKOV3-Luc cells were injected with TEK-U937), NC-U937 group (SKOV3-Luc cells were injected with NC-U937), and control group (SKOV3-Luc were injected alone). There were six mice in each group.

For long-term experiments to assess tumour growth, $1 \times 10^{5}$ TEK-U937 or NC-U937 cells were injected intravenously through the tail vein every 3 days after the third day of the orthotopic injection. For the control group, $200 \mu \mathrm{l}$ RPMI1640 media was injected each time. For IGF1 block analysis, the anti-IGF1 antibody (ab9572, Abcam) was used every 3 days after the third day of the orthotopic injection through intraperitoneal injection.

After 4 weeks, following euthanasia, the left ovary and enterocelia of the regions showing clear luciferase signal were dissected. Tumour total flux, tumour weight, and the distribution of the tumour were recorded. Tumour tissues were fixed in $4 \%$ neutral buffered formalin for frozen slide preparation.

Statistics analysis. The one-way analysis of variance (ANOVA, for all groups), $t$-test, Pearson Chi-Square test and Mann-Whitney test were used to compare groups statistically using the SPSS19.0 statistical software package. Each test for each experimental group was shown in results. The correlation between groups was assessed by Pearson's analysis. Overall survival was calculated using the Kaplan-Meier method. All tests were two-tailed, and a $P$-value of less than 0.05 was considered statistically significant.

\section{RESULTS}

TEMs amplified in EOC patients as EOC biomarker and inform the overall survival. Ovarian tissue slides from 124 EOC patients and 75 patients with benign ovarian cysts were evaluated by immunofluorescence for the presence of total tissue macrophages and TEMs (Figure 1A). The number of total tissue macrophages identified as $\mathrm{CD}^{+} 8^{+}$cells is significantly higher $(P<0.001$, student's $t$-test, Figure 1B) in EOC patients' ovarian tissue $(8.12 \pm 10.89)$ than in benign cysts $(1.53 \pm 2.60)$. The number of $\mathrm{CD} 8^{+} \mathrm{Tie}^{+}$TEMs in EOC patients $(1.89 \pm 2.85)$ is also significantly higher $(P<0.001$, student's $t$-test, Figure $1 C)$ as compared with the benign cysts patients $(0.26 \pm 0.50)$. Moreover, the ratio of TEMs/total tissue macrophages in EOC patients' 

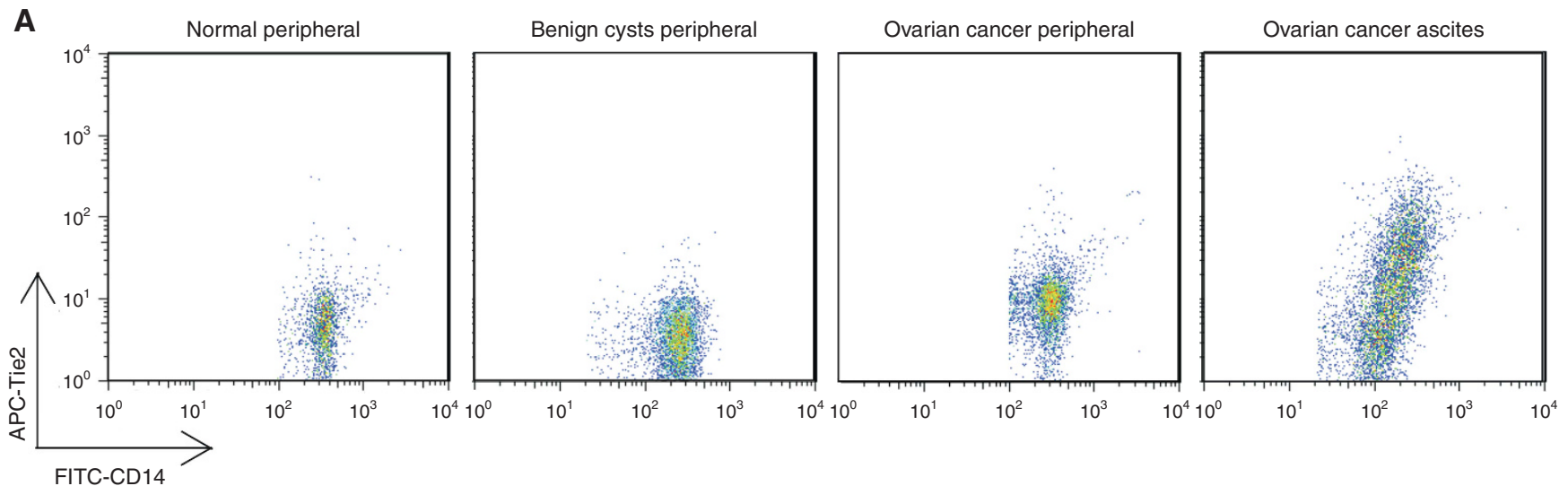

B

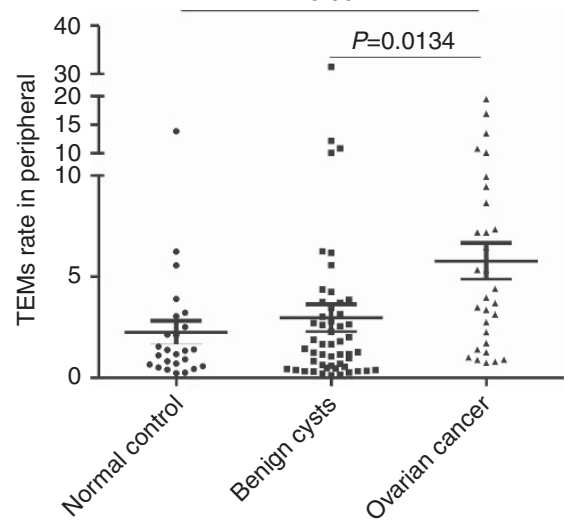

E

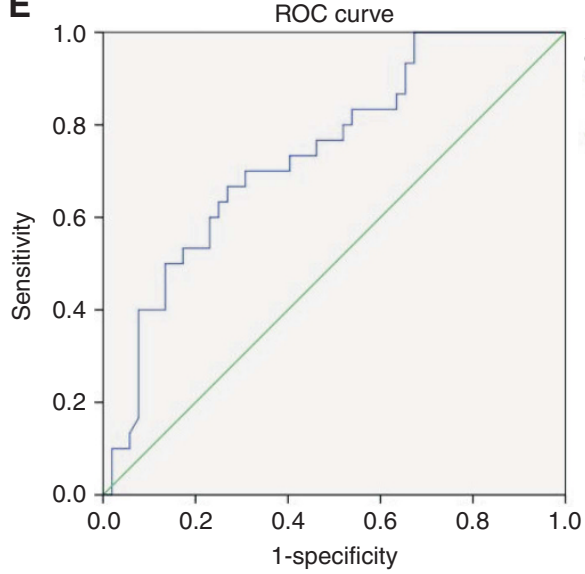

C

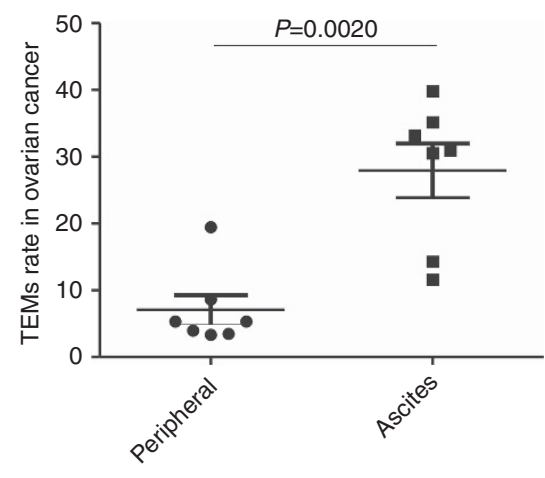

D

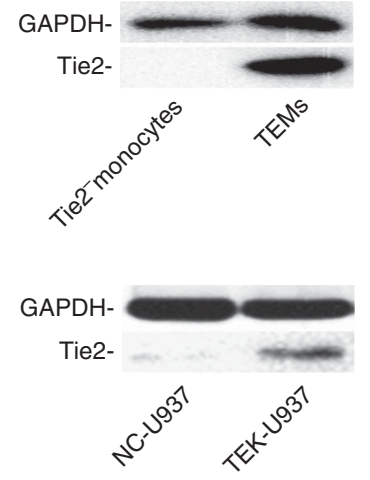

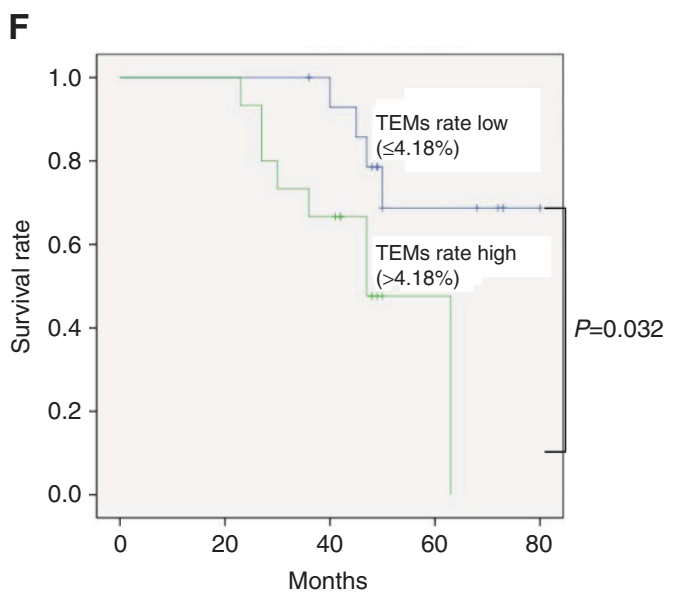

Figure 2. Increased TEMs in peripheral as a biomarker for EOC and inform the patients' outcome. (A) The CD14 ${ }^{+} \mathrm{Tie}^{+}{ }^{+}$TEMs rate in total CD14 ${ }^{+}$monocytes was detected by flow cytometry in peripheral of healthy female donors $(n=25)$, benign ovarian cysts patients $(n=52)$, EOC patients $(n=30)$ and ascites of EOC patients $(n=7)$. (B) TEMs rate in peripheral of EOC patients was significantly higher compared with benign ovarian cysts patients and healthy control (student's t-test). (C) TEMs rate in ascites compared with matched peripheral blood of the same EOC patients (student's t-test). (D) Tie2 expression was detected in primary TEMs and TEK-overexpressing monocyte TEK-U937 by WB. (E) ROC analysis was performed in order to assess the diagnostic value of TEM rate in peripheral for differentiating EOC $(n=30)$ from benign ovarian cysts $(n=52)$. (F) In EOC patients, the overall survival rate was compared between patients with TEMs rate high (TEMs rate in peripheral $>4.18 \%, n=15)$ and TEMs ratio low (TEMs ratio $\leqslant 4.18 \%, n=15)$ using the Kaplan-Meier method, with the log-rank test for comparison.

ovarian tissue $(25.78 \pm 13.05 \%)$ is also significantly higher $(P<0.001$, student's $t$-test, Figure 1D) than in benign cysts patients $(25.78 \pm 13.05 \%)$. Similar results were found in 15 peritoneum slides obtained from ovarian cancer patients and 10 peritoneum slides of benign ovarian cysts patients. Both TAMs $(10.57 \pm 4.50$ vs $1.60 \pm 2.09, \quad P<0.0001, \quad t$-test $)$ and TEMs $(1.93 \pm 1.14$ vs $0.26 \pm 0.31, \quad P=0.0002$, student's $t$-test $)$ were increased in peritoneum of ovarian cancer patients (Supplementary Figure 1A-C). Immunofluorescence staining of
Tie2 together with CD31 in blood vessels of ovarian cancer tissue showed Tie2 is also expressed in the vessels (Supplementary Figure 4A).

The ovarian tissue infiltrated TEMs number and ratio at different levels to determine the most optimal cutoff value to predict the ovarian tumour as benign cyst or EOC was evaluated. The ROC curve analyses showed that the TEMs number of 1.9 yielded the most optimal predictive value with the area under the curve (AUC) as 0.833 (95\% CI, 0.776 to $0.891, P<0.0001)$. And the 
A

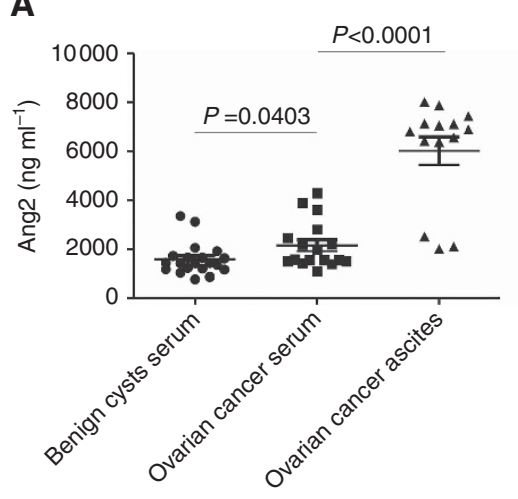

B

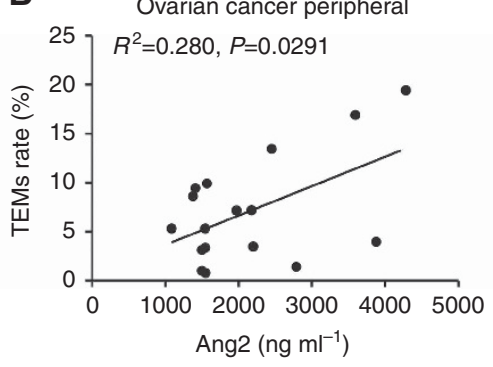

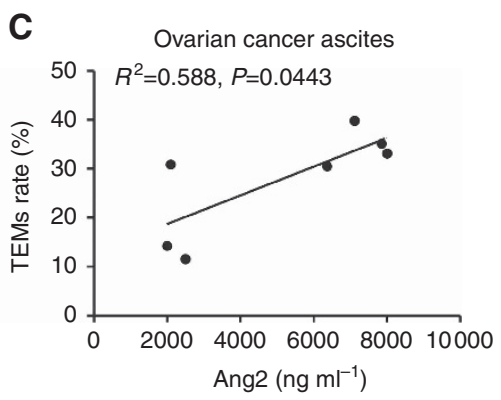

D
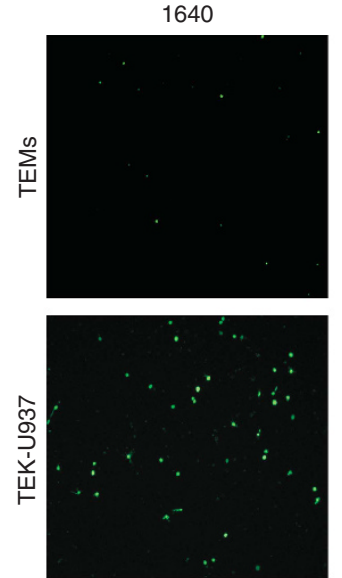

1640
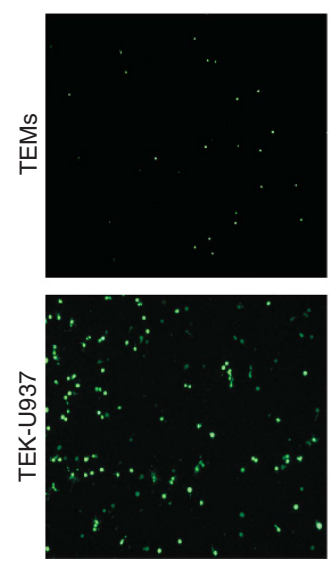

E

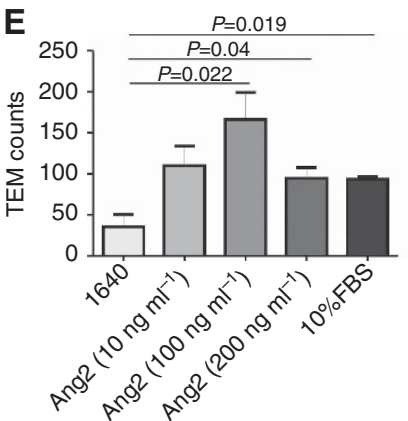

Ang2 (10 $\left.\mathrm{ng} \mathrm{ml}^{-1}\right)$
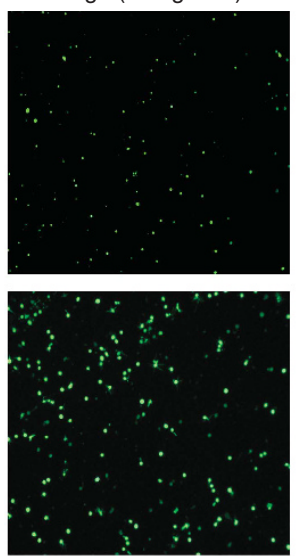

Ang2 (100 $\mathrm{ng} \mathrm{ml}^{-1}$ )
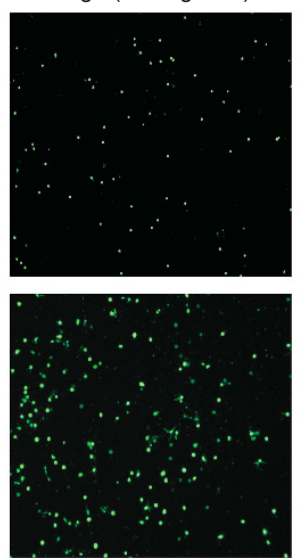

Ang2 (100 $\left.\mathrm{ng} \mathrm{ml}^{-1}\right)$
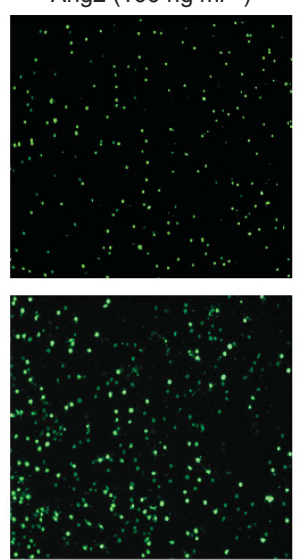

Heated-Ang2
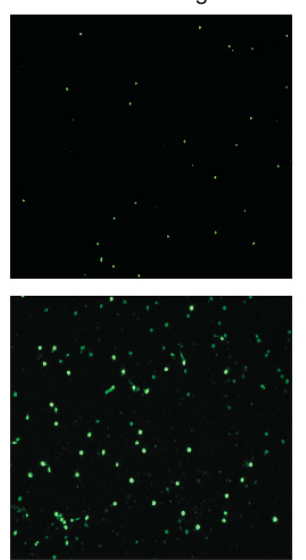

Ang2 (200 $\left.\mathrm{ng} \mathrm{ml}^{-1}\right)$
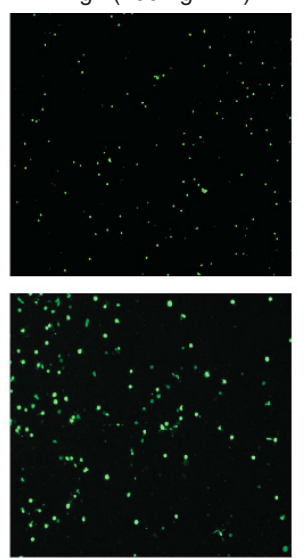

Ang2+anti-Tie2
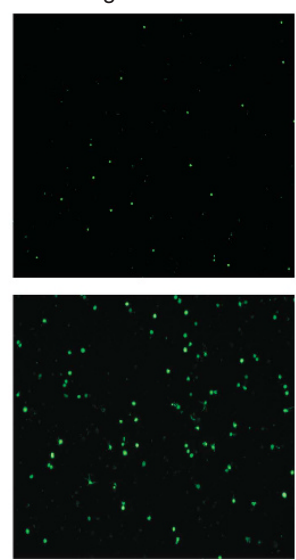

$10 \%$ FBS
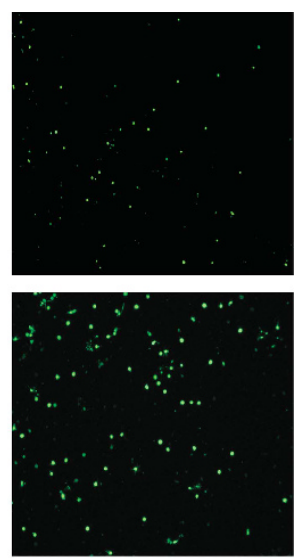

$10 \%$ FBS
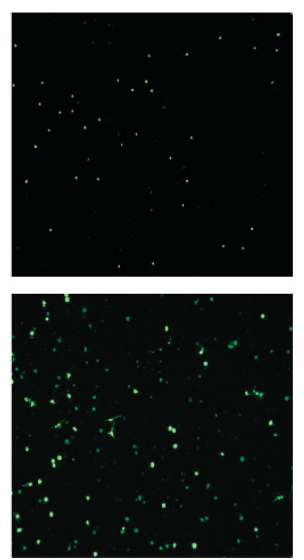
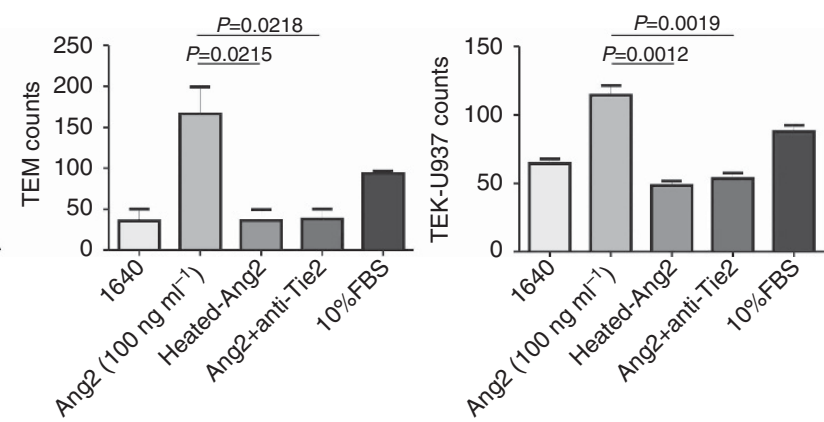

Figure 3. Increased Ang2 recruited TEMs and TEK-U937. (A) The Ang2 level was significantly higher in the ascites $(n=14)$ and serum ( $n=17)$ of EOC patients compared with the serum of benign ovarian cysts patients ( $n=20$, student's t-test) according to the ELISA assay. (B and C) Increased Ang2 was positively correlated to the TEMs rate in peripheral and ascites of EOC patients. (D) The migration ability of TEMs or TEK-U937 to medium alone (1640) or medium containing various doses of Ang2 from 10 to $200 \mathrm{ng} \mathrm{ml}^{-1}$ or $10 \% \mathrm{FBS}$ and the Ang2-Tie2 blocking assays were conducted. (E) The migration was significantly enhanced by Ang2 at the dose of $100 \mathrm{ng} \mathrm{ml}^{-1}$ compared with 1640 as a negative control and heat-inactivation of Ang2 or treatment with neutralising anti-Tie2 antibodies inhibited cell migration in response to Ang2. 

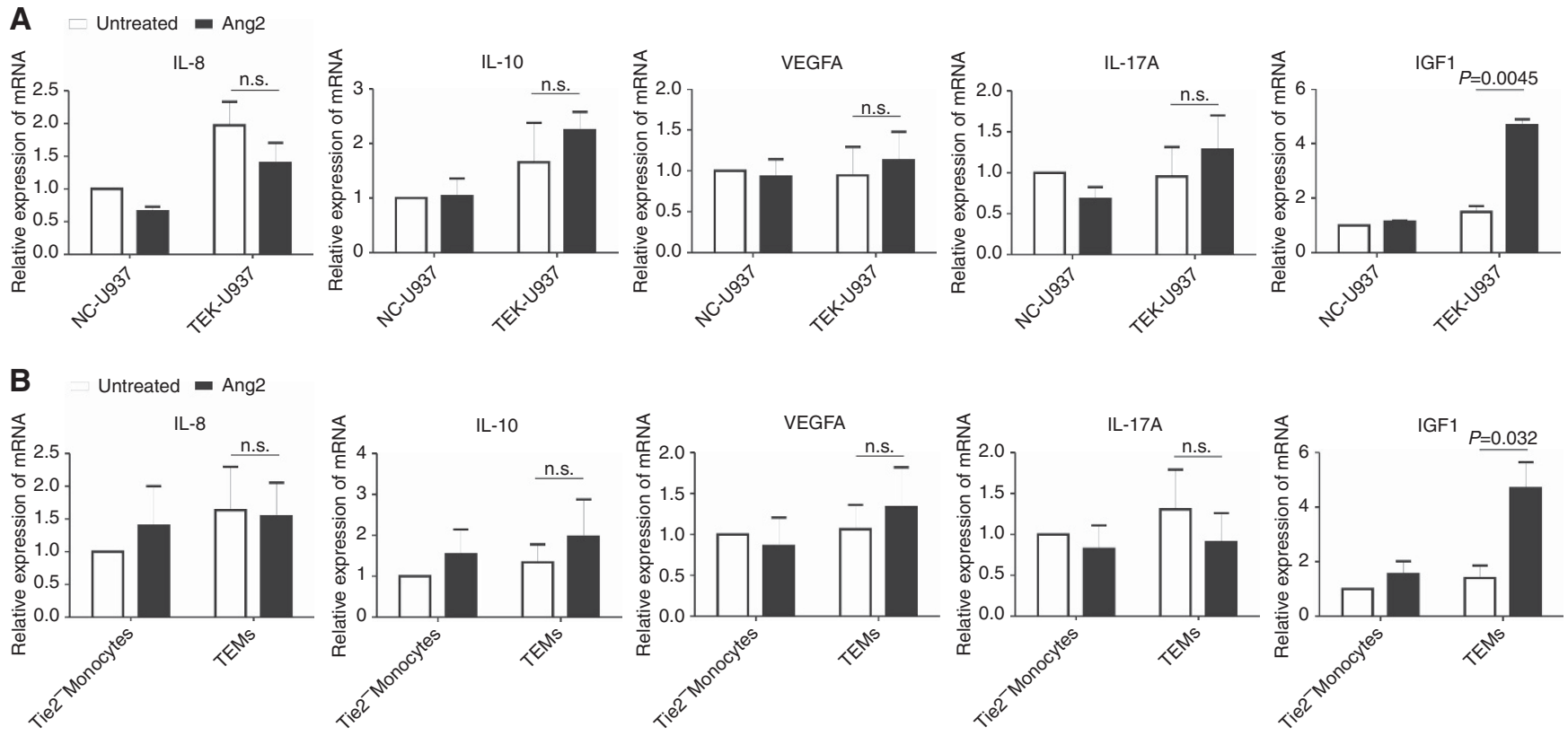

C

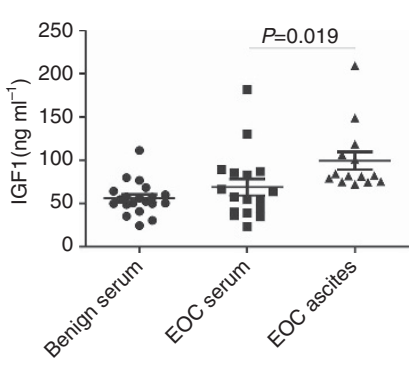

D

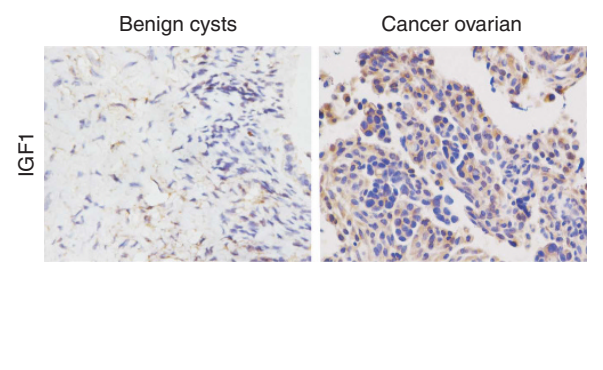

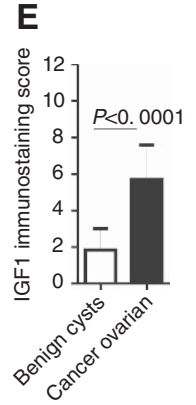

Figure 4. Increased Ang2 enhanced the IGF1 secretion of TEMs and TEK-U937. (A and B) Ang2 stimulation increased the IGF1 expression in TEKU937 $(n=3)$ and primary TEMs ( $n=3$, student's t-test). (C) IGF1 level was significantly increased in ascites of EOC patients $(n=14)$ compared with the serum of EOC patients $(n=17)$ and benign ovarian cysts patients $(n=20$, student's $t$-test) according to the ELISA assay. (D) IGF1 level in ovarian tissues of EOC patients $(n=124)$ and benign ovarian cysts patients $(n=75)$ was detected by immunohistochemistry. (E) The IGF1 expression is significantly higher in EOC ovarian tissues compared with benign ovarian cysts patients (student's $t$-test).

TEMs/total tissue macrophage ratio $5.76 \%$ the most optimal predictive value with the AUC as 0.800 (95\% CI, 0.730 to 0.869 , $P<0.0001$, Figure 1E). The median TEMs number in EOC ovarian tissue (TEMs number $=1.3$ ) was determined as the cutoff value of TEMs low group and TEMs high group. The EOC patients with high TEMs infiltrated number in ovarian tissue exhibited a significantly shorter overall survival time $(49.37 \pm 3.89)$ compared with those with low TEMs infiltrated number (72.94 \pm 3.16 , $P<0.0001$, Log-Rank test, Figure 1F, Table 1). Also, the median TEMs/total tissue macrophage ratio in EOC ovarian tissue (TEMs ratio $=24.2 \%$ ) was determined as the cutoff value of TEMs ratio low group and TEMs ratio high group. The EOC patients with high TEMs ratio exhibited a significantly shorter overall survival time (53.54 \pm 3.65$)$ compared with those with low TEMs ratio (66.72 $\pm 3.65, P<0.029$, Log-Rank test, Figure $1 \mathrm{G})$.

Moreover, the rate of TEMs (Tie ${ }^{+} \mathrm{CD} 14^{+}$) in monocytes $\left(\mathrm{CD} 14^{+}\right)$was evaluated in the peripheral blood of 25 female healthy donors, 52 benign cysts patients, and 30 EOC patients using flow cytometry (Figure 1A). The TEMs rate $(5.77 \% \pm 4.86 \%)$ in the peripheral blood in EOC patients was significantly higher ( $P=0.0024 v s$ healthy controls, $P=0.0124 v s$ benign ovarian cysts, Student's $t$-test, Figure 2B) than in healthy controls $(2.25 \% \pm 2.89 \%)$ and patients with benign ovarian cysts $(2.96 \% \pm 4.85 \%)$. The TEMs rate in ascites $(27.90 \% \pm 10.72 \%)$ from seven EOC patients was also evaluated (Figure $2 \mathrm{~A}$ ) and the frequency in ascites was significantly higher $(P=0.002$, paired $t$ test, Figure 2C) than that of matched peripheral blood samples $(7.07 \% \pm 5.75 \%)$ in each patient. The Tie2 expression in primary TEMs isolated by FACS and in the TEK over-expression monocytes (TEK-U937) were confirmed by WB (Figure 2D). The gating strategy and the isotype used in the flow cytometry were shown in Supplementary Figure 2 and the TEK over-expression in TEK-U937 was also measured by fluorescent microscope and FACS detection of GFP (Supplementary Figure 3).

The peripheral TEMs rate at different levels to determine the most optimal cutoff value to predict the ovarian tumour as benign cyst or EOC was evaluated. The ROC curve analysis showed that the TEMs rate in peripheral of $3.08 \%$ yielded the most optimal predictive value with the AUC as 0.74 (95\% CI, 0.631 to 0.849 , $P<0.0001$, Figure 2E). The median peripheral TEMs rate in EOC patients (TEMs rate $=4.18 \%$ ) was determined as the cutoff value of TEMs rate low group and TEMs rate high group. The EOC patients with high peripheral TEMs rate exhibited a significantly shorter overall survival time $(48.49 \pm 4.40)$ compared with those with low peripheral TEMs rate $(69.34 \pm 4.50, P=0.032$, Log-Rank test, Figure 2F).

Increased Ang2 recruited primary TEMs and TEK-U937. The concentration of Ang2, as the ligand of Tie2, was examined by ELISA in serum samples of 17 EOC patients, 20 benign cysts 
A

RPMI1640

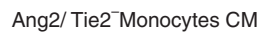

Ang2/TEMs CM

IGF1

Ang2/TEMs CM

IGF1
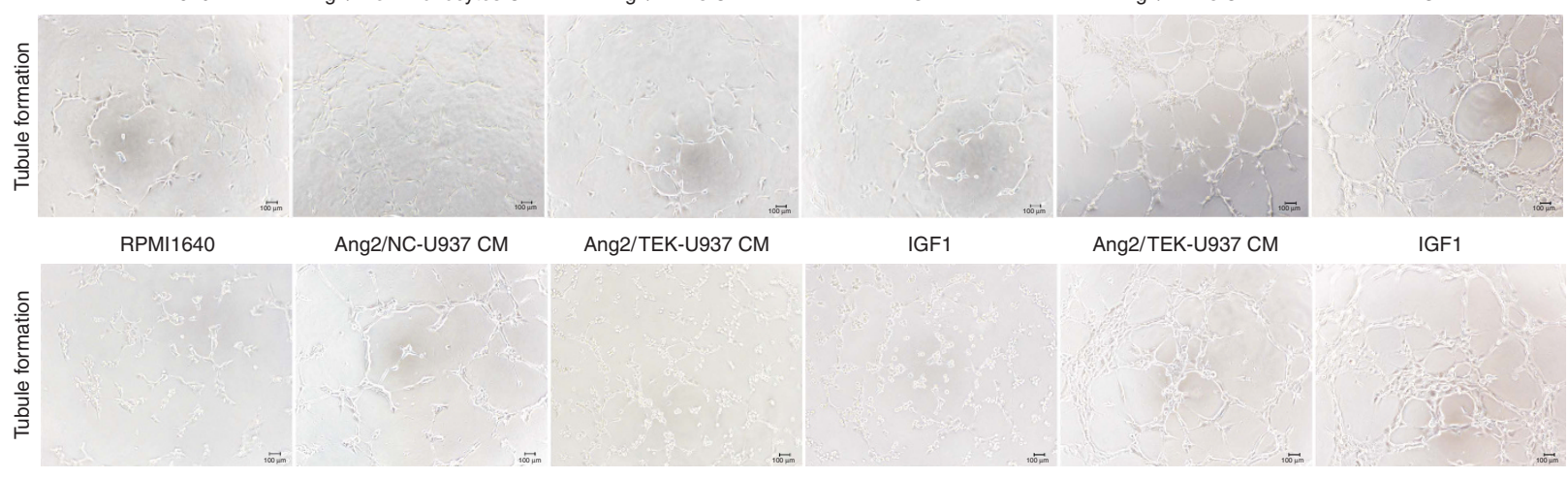

Anti-IGF1

B

$P=0.0022$ $P=0.0082$ $P=0.0060$
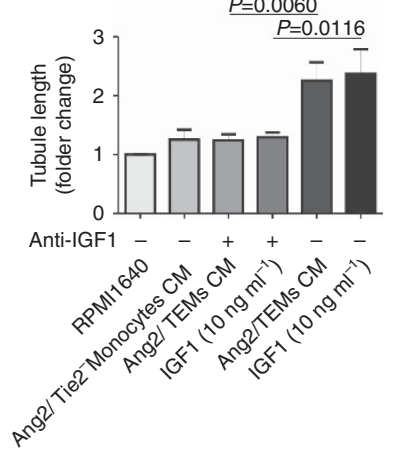

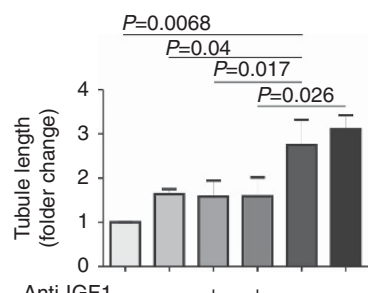

Anti-IGF1 -

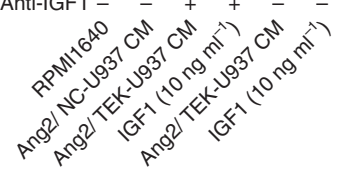

C

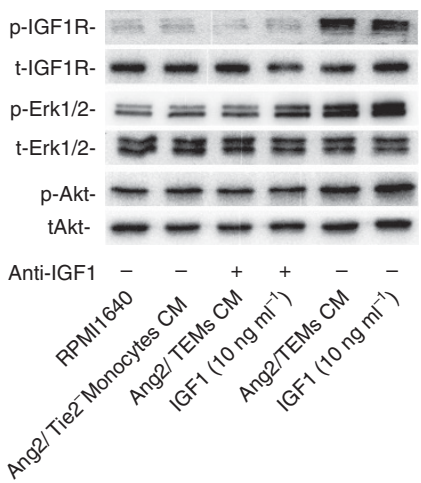

D

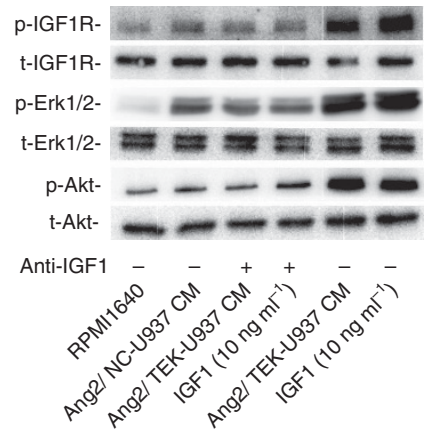

Figure 5. Ang2 enhanced the TEMs and TEK-U937 promoting tubule formation via IGF1 pathway in vitro. (A) HUVEC tubule formation assay in vitro was conducted. (B) The Ang2 stimulated primary TEMs or TEK-U937 conditioned medium (CM) induced significantly more tubule formation by calculating the tubule length ( $n=3$ in each group, student's $t$-test). ( $C$ and $\mathbf{D}$ ) The Ang2 stimulated primary TEMs or TEK-U937 conditioned medium (CM) induced the phosphorylation of IGF1R, Akt and Erk1/2 detected in HUVECs by WB.

patients, and ascites samples of 14 EOC patients (Figure 3A). Ang2 levels in the serum of EOC patients $\left(2142 \pm 958.6 \mathrm{ng} \mathrm{ml}^{-1}\right)$ were significantly higher $(P=0.0403$, student's $t$-test $)$ than benign cysts patients $\left(1575 \pm 651.5 \mathrm{ng} \mathrm{ml}^{-1}\right)$. Moreover, Ang2 level in the ascites of EOC patients $\left(6013 \pm 2123 \mathrm{ng} \mathrm{ml}^{-1}\right)$ was significantly higher than that in the patient-matched serum samples $(P<0.001$, student's $t$-test) in each patient. The increased Ang2 was positively correlated to the TEMs rate in peripheral $\left(R^{2}=0.280, P=0.0291\right)$ and ascites of EOC patients $\left(R^{2}=0.588, P=0.0443\right.$, Figure $3 \mathrm{~B}$ and $\left.\mathrm{C}\right)$.

The effects of Ang2 on the migration of human TEMs and TEKU937 were further examined (Figure 3D). We found that Ang2 induced significant migration of both TEMs and TEK-U937, with the highest response corresponding to an Ang2 concentration of $100 \mathrm{ng} \mathrm{ml}^{-1}$ (Figure 3E, $P=0.022$ or 0.0008 vs RPMI1640 control). To confirm that the observed chemotactic response was induced by specific interactions between Ang2 and Tie2, TEMs or TEK-U937 were pretreated with neutralising anti-Tie2 antibodies. Anti-Tie2 antibodies or heat inactivation of Ang2 significantly blocked Ang2-induced cell migration (Figure 3E).

Ang2 induced IGF1 increasing in primary TEMs and TEKU937. The effects of Ang2 on cytokine expression in TEMs and TEK-U937 were evaluated by qPCR before and after treatment with Ang2 (Figure 4A and B). The qPCR results showed that IGF1 was significantly increased in both TEMs $((P=0.032)$ and TEKU937 cells after treatment $(P=0.0045$, student's $t$-test), whereas the mRNA levels of interleukin-8 (IL-8), interleukin-10 (IL-10), vascular endothelial growth factor-A (VEGFA), and interleukin17A (IL-17A) were not changed. Anti-Tie2 antibodies significantly reserved the IGF1 increased in TEMs and TEK-U937 induced by
Ang2 (Supplementary Figure 5). Moreover, ELISA assays showed that the IGF1 levels in ascites of the EOC patients was significantly higher than in serum $(P=0.019$, student's $t$-test, Figure $4 C)$. Also, IGF1 expression level was significantly increased in 124 EOC patients compared with 75 benign cysts patients as evidenced by a significantly higher immunostaining score $(P<0.0001$, student's $t$-test, Figure $4 \mathrm{D}$ and E). Moreover co-immunostaining of IGF1 and TEMs in ovarian tissues of EOC was performed by immunofluorescence and the TEMs expressed high IGF1 level was observed (Supplementary Figure 4B).

TEMs promote angiogenesis via IGF1. Primary TEMs or TEK-U937 cells were firstly stimulated by Ang2 (100 $\mathrm{ng} \mathrm{ml}^{-1}$ ) for $24 \mathrm{~h}$ and the conditioned medium (CM) was collected for assays of endothelial tubule formation (Figure 5A). IGF1 with the concentration of $10 \mathrm{ng} \mathrm{ml}^{-1}$ served as the positive control. The results showed that Ang2-stimulated primary TEMs or TEK-U937 cells CM significantly increased capillary tubule formation compared with the Ang2-stimulated-NC CM (Figure 5B, $P=0.04$, student's $t$-test). Addition of an IGF1 inhibiting antibody significantly reversed tubule formation induced by Ang2-stimulated TEMs or TEK-U937 cells CM (Figure 5B, $P=0.017$, student's $t$-test). The phosphorylation status of IGF1R, Erk1/2 and Akt in HUVECs were significantly increased after stimulation with Ang2stimulated TEMs or TEK-U937 CM and addition of anti-IGF1 blocked the phosphorylation of these proteins in HUVECs (Figure 5C and D). After TEMs or TEK-U937 pre-treated with anti-Tie2 blocking antibody, the Ang2-stimulated TEMs or TEKU937 CM induced phosphorylation of IGF1-IGF1R pathway proteins in HUVECs was reserved (Supplementary Figure 5). 
A

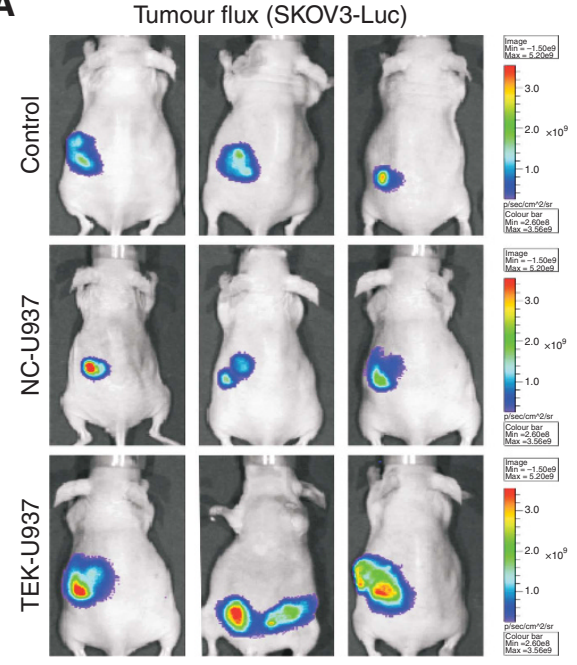

E

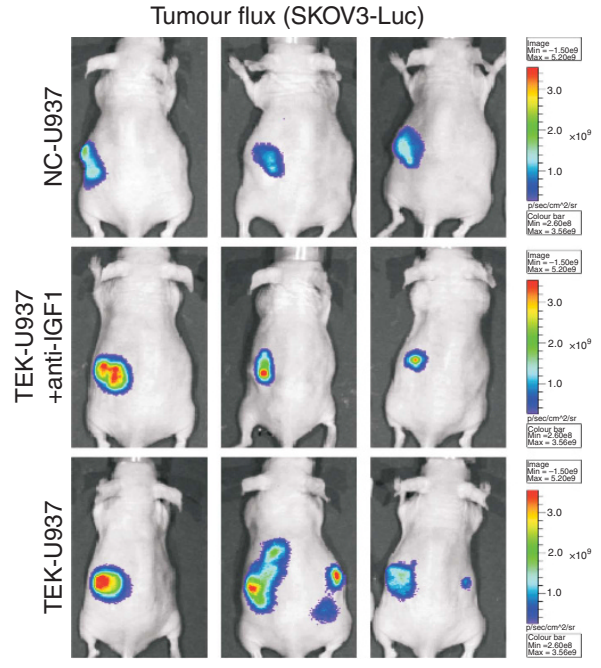

B

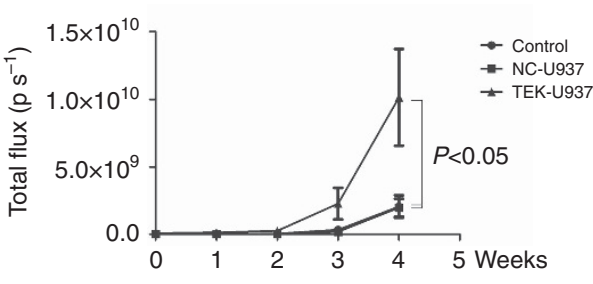

C

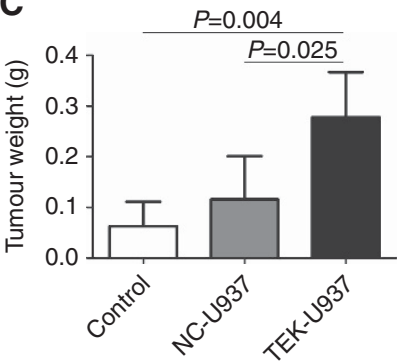

D

Tumour metastasis organs and numbers (number of metastasis mice/total mice in each group)

\begin{tabular}{lcccccc}
\hline Group & Ovarian-R & Uterine & L.N. & Spleen & Peritoneum & Total metastasis organs \\
\hline Control & $0 / 6$ & $0 / 6$ & $0 / 6$ & $0 / 6$ & $4 / 6$ & 4 \\
NC-U937 & $1 / 6$ & $0 / 6$ & $1 / 6$ & $0 / 6$ & $3 / 6$ & 5 \\
TEK-U937 & $3 / 6$ & $0 / 6$ & $2 / 6$ & $1 / 6$ & $6 / 6$ & 12 \\
\hline
\end{tabular}

Pearson Chi-square test, $P=0.02$ TEK-U937 vs control group, $P=0.045$ TEK-U937 vs NC-U937 group

F

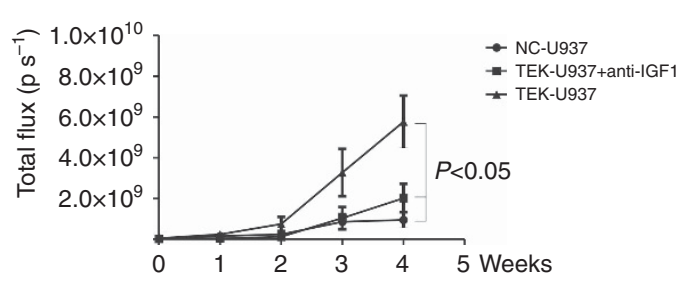

G

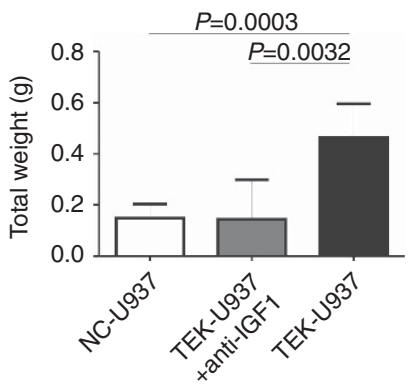

H Tumour metastasis organs and numbers (number of metastasis mice/total mice in each group)

\begin{tabular}{lcccccc} 
Group & Ovarian-R & Uterine & L.N. & Intestine & Peritoneum & Total metastasis organs \\
\hline NC-U937 & $0 / 6$ & $0 / 6$ & $1 / 6$ & $0 / 6$ & $1 / 6$ & 2 \\
TEK-U937 & $1 / 6$ & $0 / 6$ & $2 / 6$ & $0 / 6$ & $1 / 6$ & 4 \\
+anti-IGF1 & $2 / 6$ & $1 / 6$ & $1 / 6$ & $1 / 6$ & $6 / 6$ & 11 \\
TEK-U937 & $2 / 6$ & $1 / 6$ & \\
\hline
\end{tabular}

Pearson Chi-square test, $P=0.005$ TEK-U937 vs NC-U937group, $P=0.037$ TEK-U937 vs TEK-U937+anti-IGF1 group

Figure 6. TEK-U937-promoted tumour development and metastasis in vivo. (A) SKOV3-Luc expression was detected by BLI in the tumour burden mouse model. (B and C) The TEK-U937 group showed significantly higher flux and tumour weight compared with the NC-U937 and control groups ( $n=6$ in each group, Mann-Whitney test). (D) TEK-U937 significantly promoted tumour metastasis compared to the NC-U937 and control groups (Pearson Chi-square test). (E-H) IGF1 blocking antibody reversed the increased tumour flux, tumour weight and tumour metastasis promoted by TEK-U937 $(n=6)$.

TEK-U937 promote angiogenesis and progress of EOC in vivo. TEK-U937 cells behaved highly similar to primary TEMs in vitro, and was used for further study in vivo. Tumour development occurred in $100 \%$ of orthotopically injected ovaries in mice and longitudinal bioluminescence imaging for fluorescent tumour burden was assessed (Figure 6A). The test of BLI signal intensity confirmed that in the TEK-U937 group $\left(2.27 \times 10^{9} \pm 2.85 \times 10^{9}\right.$ by week $3,1.01 \times 10^{10} \pm 8.77 \times 10^{9}$ by week 4), tumour growth (total flux, $\mathrm{ps}^{-1}$ ) was enhanced compared with the NC-U937 group $\left(1.98 \times 10^{8} \pm 1.92 \times 10^{8}\right.$ by week 3 , $1.97 \times 10^{9} \pm 1.63 \times 10^{9}$ by week 4) and the control group $\left(3.26 \times 10^{8} \pm 3.93 \times 10^{8}\right.$ by week $3,2.05 \times 10^{9} \pm 2.05 \times 10^{9}$ by week $4, n=6$ in each group, Figure $6 \mathrm{~B})$. The tumour weight $(\mathrm{g})$ of the TEK-U937 group $(0.28 \pm 0.09 \mathrm{~g})$ was also much higher at the time of killing than that of the NC-U937 $(0.12 \pm 0.09 \mathrm{~g})$ and control groups $(0.06 \pm 0.05 \mathrm{~g}$, Figure $6 \mathrm{C})$.

Within the TEK-U937 group, tumour metastases were found in the contra lateral ovary, lymph nodes, spleen and peritoneum. Moreover, the peritoneum metastases were found in all the six mice of TEK-U937 group. There are significantly more tumour metastases in TEK-U937 group compared with the NC-U937 group ( $P=0.045$, Pearson Chi-square test) and the control group $(P=0.004$, Pearson Chi-square test, Figure 6D).

To evaluate tumour angiogenesis, MVD was calculated by anti-CD31 staining in murine tumour tissues and the mice peritoneum (Figure 7A). MVD was significantly higher in ovarian tumour tissue of TEK-U937 group (23.70 \pm 4.78$)$ compared with in $\mathrm{NC}$-U937 group $(14.63 \pm 2.94, P=0.003$, student's $t$-test) and in control group $(13.83 \pm 2.85, P=0.001$, student's $t$-test, Figure 7B). Furthermore, MVD was significantly higher in peritoneum of TEK-U937 group (27.27 \pm 6.48$)$ compared with in NC-U937 group $(20.37 \pm 1.52 P=0.029$, student's $t$-test $)$ and in control group $(15.13 \pm 1.68, P=0.001$, student's $t$-test, Figure 7C).

Further study of IGF1 blocking was performed and anti-IGF1 blocking antibody significantly reversed the tumour growth, tumour metastases and the angiogenesis promoted by TEK-937 in vivo (Figures $6 \mathrm{E}-\mathrm{H}, 7 \mathrm{D}-\mathrm{F}$ ).

Immunofluorescence detection of GFP was performed and TEK-U937-GFP cells and NC-U937-GFP cells were homogeneously distributed in the mice tumour tissue but not in the control group (Supplementary Figure 6). 
A

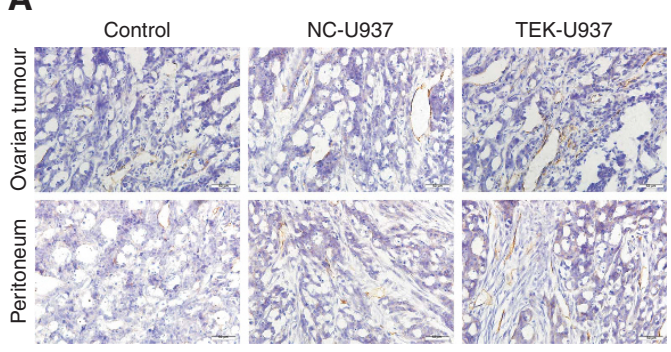

D

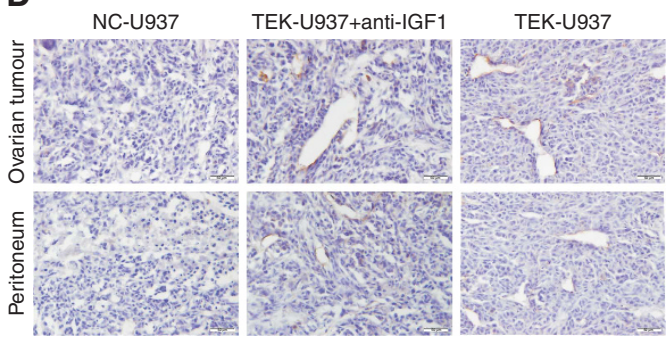

B

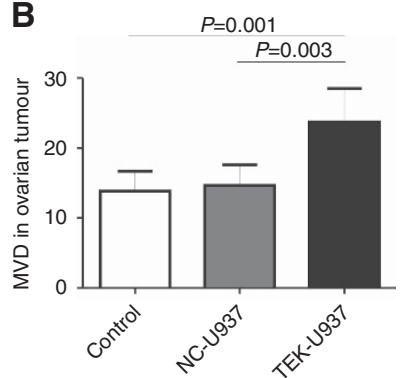

$\mathbf{E}$

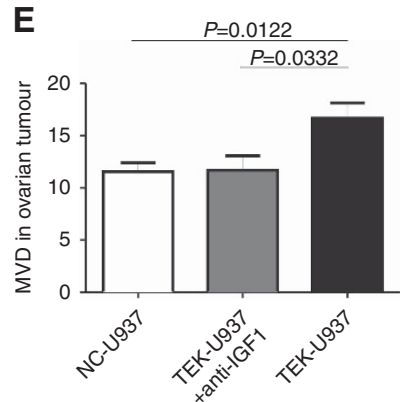

C

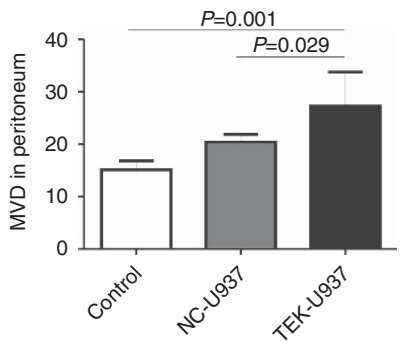

$\mathbf{F}$

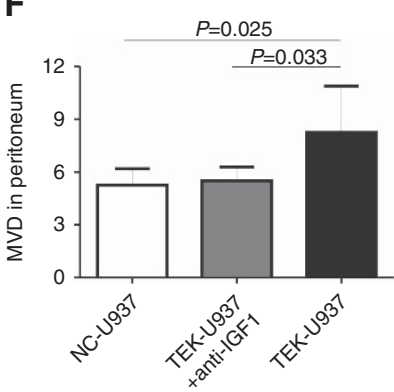

Figure 7. TEK-U937-promoted tumour angiogenesis in vivo. (A) MVD value was calculated in mice tumour tissues and the corresponding peritoneum by immunohistochemistry staining of CD31 $(n=6)$. (B and C) The MVD value of TEK-U937 group is significantly increased in tumour local and corresponding peritoneum (Mann-Whitney test). (D-F) IGF1 blocking antibody reversed the angiogenesis promoted by TEK-U937 $(n=6)$.

A

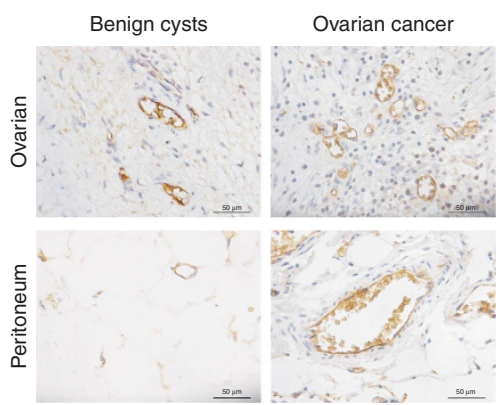

B

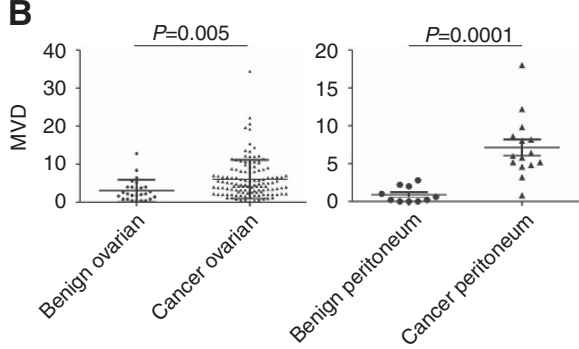

C
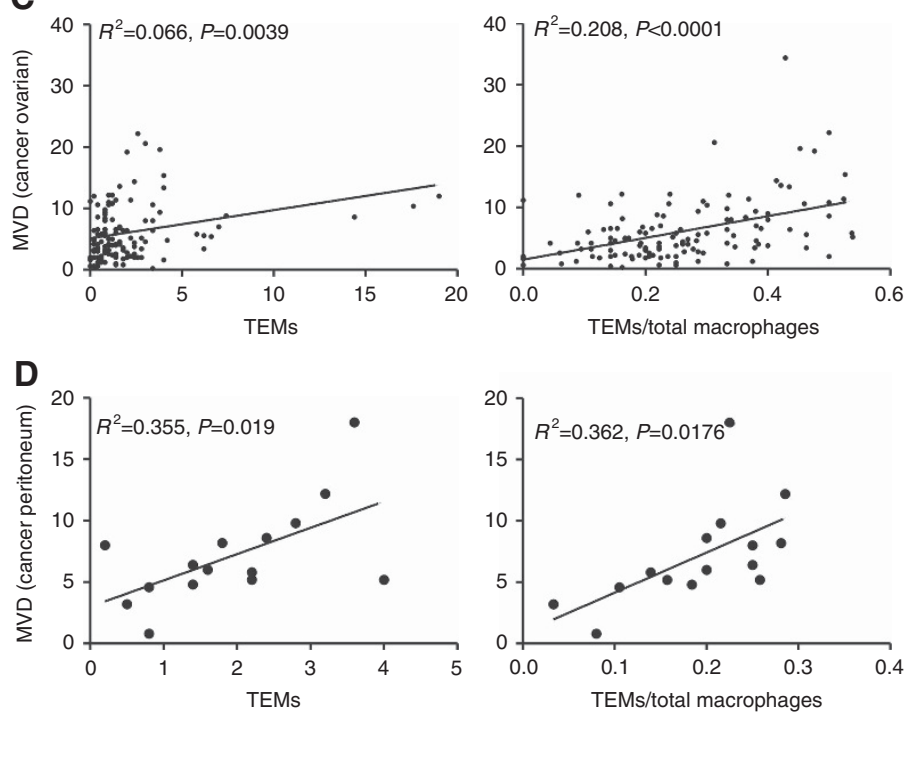

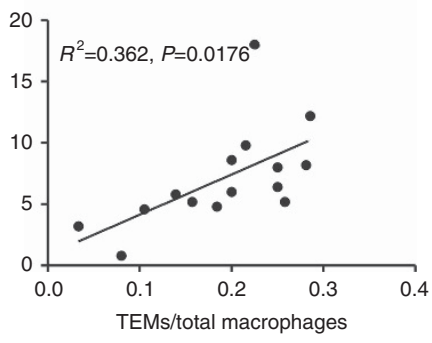

Figure 8. TEMs correlate with MVD in ovarian cancer tissue. (A) MVD value was calculated in ovarian tumour tissues of EOC patients ( $n=124)$, ovarian tumour tissues of patients with benign ovarian cysts $(n=75)$, the peritoneum of EOC patients $(n=15)$ and peritoneum of patients with benign ovarian cysts $(n=10)$. (B) The MVD values in both ovarian tissues and peritoneum of the EOC patients are significantly increased compared with the patients with benign ovarian cysts (student's t-test). (C and D) The increased MVD values in EOC patients' ovarian tissues and peritoneum were positively correlated to the infiltrated TEMs number and the TEMs/total macrophages ratio.

TEMs correlate with MVD in EOC patients. To determine if infiltrated TEMs correlate with tumour angiogenesis, we measured MVD using anti-CD31 staining in ovarian tissue slides obtained from 124 ovarian cancer patients and 75 patients with benign cysts (Figure $8 \mathrm{~A}$ ). As expected, MVD in ovarian cancer patients $(6.05 \pm 5.08)$ was significantly higher (Figure $8 \mathrm{~B}$, $P<0.0001$, student's $t$-test) than in patients with benign cysts
(1.91 \pm 2.21$)$. microvascular density in the perineum of 15 ovarian cancer patients $(7.02 \pm 4.09)$ was also significantly higher (Figure $8 \mathrm{~B}, P=0.0001$, student's $t$-test) than in 10 benign cysts patients $(0.90 \pm 1.06)$. Furthermore, MVD values in both ovarian cancer tissues and peritoneum were positively correlated with the TEMs number and TEMs/total macrophages ratio (Figure $8 \mathrm{C}$ and $\mathrm{D}$ ). 


\section{DISCUSSION}

TEMs, a novel subset of monocytes, are considered important not only for the development of blood-vessel formation in tumours but also for other human diseases, such as ischemic limb (Patel et al, 2013). Previous studies have shown that monocytes are the principal population of mononuclear cells that express Tie2 in the circulation system, and one study has shown that selective elimination of TEMs impairs angiogenesis and tumour growth in a tumour-burden mouse model (De Palma et al, 2007). Thus, TEMs have been discussed as a potential therapeutic target to inhibit angiogenesis in tumours (Daly et al, 2013).

The data presented here show that both circulating TEMs and TEMs infiltrated into ovarian tissues and peritoneum are significantly increased in EOC patients compared with control groups. Moreover, the frequency of TEMs was significantly increased in ascites compared to matched samples of peripheral blood in EOC patients. ROC and survival analysis found that the ovarian tissue infiltrated TEMs number, the TEMs/total macrophages ratio in tissue, together with the TEMs rate in patients peripheral, could serve as novel biomarkers and prognosis predictors of EOC and particular EOC-derived factors may promote the recruitment of TEMs.

CCR2, the receptor for the chemokine MCP1 that plays an important role in the recruitment of monocytes, was not expressed in TEMs (Augustin et al, 2009), suggesting that TEMs might be attracted to tissues by other factors. Ang2, as a ligand for Tie2, is upregulated in malignant tumours as a response to hypoxia and functions as a chemoattractant for human TEMs (Huang et al, 2011). Interestingly, Ang2 levels were increased in the ascites fluid of EOC patients and were significantly higher than matched sets of serum. Increased Ang2 levels in EOC patients, especially in the ascites samples, may play an important role in recruiting TEMs to tissue. Pretreatment of cells with anti-Tie2 antibody reversed TEMs attraction by Ang2. These results suggest that TEMs migration into tumour tissue may be regulated by increased Ang2 levels in the tumour microenvironment and expression of Tie2 on TEMs. Similar results were observed in TEK-U937 cell line, supporting the hypothesis that the over-expressed Tie 2 in monocytic cell lines interact with the ligand Ang2. These results also suggest that TEKU937 may be used as a Tie2 expressing monocytic cell model in future studies.

As classical core molecules in tumour angiogenesis, Ang2-Tie2 interactions in endothelial cells were hypothesised to induce the abnormal angiogenesis caused by IL6 in ovarian cancer (Gopinathan et al, 2015). In our study, high TEMs infiltrated in ovarian cancer, another prominent cell subset that expressed Tie2 molecule, might also play an important role in angiogenesis as the target cell of Ang2. The effects of Ang2 on TEMs function have been studied (Coffelt et al, 2010), and the results demonstrate that Ang2 can markedly enhance the pro-angiogenic activity of TEMs, while targeting the Ang2/Tie2 pathway could inhibit the functions of TEMs (De Palma and Naldini, 2011).

In this study, we explored the gene expression changes in TEMs before and after Ang2 stimulation. The results show that the mRNA level of the cytokines IL-8 and IL-10, IL-17A, as well as VEGFA, a classical growth factor that promotes angiogenesis, were not changed in primary TEMs or TEK-U937 by Ang2 stimulation. However, the mRNA level of IGF1 is significantly increased after Ang2 stimulation. This confirmed results observed in our previous study of microarray assays of TEMs and Tie2-Monocytes isolated from peripheral blood of healthy donors, which showed that IGF1 mRNA levels were increased in TEMs (Wang et al, 2016). These data indicate that circulating Ang2 could upregulate the expression of IGF1 in TEMs. Further studies verified that IGF1 was also significantly increased in the ascites and the ovarian tissue of the EOC patients.

The insulin-like growth factor (IGFs) family and their receptors comprise an important growth factor system that is necessary for the function of many cells under normal physiological conditions (Rother and Accili, 2000). In pathological situations, evidence has indicated that IGFs play an important role in tumourigenesis due to powerful anti-apoptotic effects (Khandwala et al, 2000). In human tumours, such as prostate cancer, a high circulating level of IGF1 is correlated with a high risk of tumour development (Cohen, 1998; Wolk et al, 1998). With respect to the function of IGF1 in angiogenesis-promoting progression, we found that capillary tubule formation in HUVECs was significantly enhanced by TEMs/TEK-U937-CM. Evidence of activation of the IGF1-IGF1R pathway in HUVECs was provided by detection of phosphorylation of IGF1R signal transduction cascade molecules, including IGF1R, Akt and Erk1/2 (LeRoith et al, 1995). The results indicate that IGF1-mediated signalling may play an important role in angiogenesis induced by the Tie 2 over-expressing monocytic cells.

In summary, we found that TEMs are significantly increased in peripheral blood, ascites, and tissue samples of ovarian cancer patients. And the increased TEMs have diagnostic value in ovarian cancer. Our study also showed that Ang2 was increased in ovarian cancer ascites, and could attract TEMs to tumour tissue. Furthermore, MVD in ovarian cancer tissue correlated with TEMs number and frequency. We also found that the TEMs promote angiogenesis via IGF1 in both in vivo and in vitro experimental systems after stimulation by Ang2. Thus, the Ang2-TEMs-IGF1 axis is a potential target for ovarian cancer therapy.

\section{ACKNOWLEDGEMENTS}

This study was supported by grants from the National Natural Science Foundation of China (81372787, 81072136, 81402042, $81602280,81772654,81602281$ ), the Natural Science Foundation of Shanghai (17411968100, 15411961600, 16ZR1427100, 2016CR4028A, 104119692000) the Shanghai Municipal Bureau of Health (20134033, 20164Y0003), Shanghai Pudong research project (PW2010D-5), the Shanghai Health System joint research project (2013ZYJB0201), the Shanghai science and technology (14140903400, 14YF1402600), the Top 100 Medical Elite in Shanghai (XBR 2011065) and Training plan for scientific research of Renji Hospital (RJZZ13-021).

${ }^{7}$ These authors contributed equally to this work.

\section{CONFLICT OF INTEREST}

The authors declare no conflict of interest.

\section{REFERENCES}

Augustin HG, Koh GY, Thurston G, Alitalo K (2009) Control of vascular morphogenesis and homeostasis through the angiopoietin-Tie system. Nat Rev Mol Cell Biol 10(3): 165-177.

Coffelt SB, Tal AO, Scholz A, De Palma M, Patel S, Urbich C, Biswas SK, Murdoch C, Plate KH, Reiss Y, Lewis CE (2010) Angiopoietin-2 regulates gene expression in TIE2-expressing monocytes and augments their inherent proangiogenic functions. Cancer Res 70(13): 5270-5280.

Cohen P (1998) Serum insulin-like growth factor-I levels and prostate cancer risk-interpreting the evidence. J Natl Cancer Inst 90(12): 876-879.

Daly C, Eichten A, Castanaro C, Pasnikowski E, Adler A, Lalani AS, Papadopoulos N, Kyle AH, Minchinton AI, Yancopoulos GD, Thurston G (2013) Angiopoietin-2 functions as a Tie2 agonist in tumor models, where it limits the effects of VEGF inhibition. Cancer Res 73(1): 108-118. 
De Palma M, Coukos G, Semela D (2013) TIE2-expressing monocytes: a novel cellular biomarker for hepatocellular carcinoma? Hepatology 57(4): 1294-1296.

De Palma M, Murdoch C, Venneri MA, Naldini L, Lewis CE (2007) Tie2expressing monocytes: regulation of tumor angiogenesis and therapeutic implications. Trends Immunol 28(12): 519-524.

De Palma M, Naldini L (2011) Angiopoietin-2 TIEs up macrophages in tumor angiogenesis. Clin Cancer Res 17(16): 5226-5232.

De Palma M, Venneri MA, Galli R, Sergi LS, Politi LS, Sampaolesi M, Naldini L (2005) Tie2 identifies a hematopoietic lineage of proangiogenic monocytes required for tumor vessel formation and a mesenchymal population of pericyte progenitors. Cancer Cell 8(3): 211-226.

De Palma M, Venneri MA, Roca C, Naldini L (2003) Targeting exogenous genes to tumor angiogenesis by transplantation of genetically modified hematopoietic stem cells. Nat Med 9(6): 789-795.

Gopinathan G, Milagre C, Pearce OM, Reynolds LE, Hodivala-Dilke K, Leinster DA, Zhong H, Hollingsworth RE, Thompson R, Whiteford JR, Balkwill F (2015) Interleukin-6 stimulates defective angiogenesis. Cancer Res 75(15): 3098-3107.

Huang H, Lai JY, Do J, Liu D, Li L, Del Rosario J, Doppalapudi VR, PirieShepherd S, Levin N, Bradshaw C, Woodnutt G, Lappe R, Bhat A (2011) Specifically targeting angiopoietin-2 inhibits angiogenesis, Tie2-expressing monocyte infiltration, and tumor growth. Clin Cancer Res 17(5): 1001-1011.

Jemal A, Bray F, Center MM, Ferlay J, Ward E, Forman D (2011) Global cancer statistics. CA: Cancer J Clin 61(2): 69-90.

Khandwala HM, McCutcheon IE, Flyvbjerg A, Friend KE (2000) The effects of insulin-like growth factors on tumorigenesis and neoplastic growth. Endocr Rev 21(3): 215-244.

LeRoith D, Werner H, Beitner-Johnson D, Roberts CT Jr (1995) Molecular and cellular aspects of the insulin-like growth factor I receptor. Endocr Rev 16(2): 143-163.

Matsubara T, Kanto T, Kuroda S, Yoshio S, Higashitani K, Kakita N, Miyazaki M, Sakakibara M, Hiramatsu N, Kasahara A, Tomimaru Y, Tomokuni A, Nagano H, Hayashi N, Takehara T (2013) TIE2-expressing monocytes as a diagnostic marker for hepatocellular carcinoma correlates with angiogenesis. Hepatology 57(4): 1416-1425.

Partanen J, Armstrong E, Makela TP, Korhonen J, Sandberg M, Renkonen R, Knuutila S, Huebner K, Alitalo K (1992) A novel endothelial cell surface receptor tyrosine kinase with extracellular epidermal growth factor homology domains. Mol Cell Biol 12(4): 1698-1707.

Patel AS, Smith A, Nucera S, Biziato D, Saha P, Attia RQ, Humphries J, Mattock K, Grover SP, Lyons OT, Guidotti LG, Siow R, Ivetic A, Egginton S, Waltham M, Naldini L, De Palma M, Modarai B (2013) TIE2- expressing monocytes/macrophages regulate revascularization of the ischemic limb. EMBO Mol Med 5(6): 858-869.

Pucci F, Venneri MA, Biziato D, Nonis A, Moi D, Sica A, Di Serio C, Naldini L, De Palma M (2009) A distinguishing gene signature shared by tumor-infiltrating Tie2-expressing monocytes, blood 'resident' monocytes, and embryonic macrophages suggests common functions and developmental relationships. Blood 114(4): 901-914.

Ribatti D (2009) The paracrine role of Tie-2-expressing monocytes in tumor angiogenesis. Stem Cells Dev 18(5): 703-706.

Rother KI, Accili D (2000) Role of insulin receptors and IGF receptors in growth and development. Pediatr Nephrol 14(7): 558-561.

Saharinen P, Eklund L, Miettinen J, Wirkkala R, Anisimov A, Winderlich M, Nottebaum A, Vestweber D, Deutsch U, Koh GY, Olsen BR, Alitalo K (2008) Angiopoietins assemble distinct Tie2 signalling complexes in endothelial cell-cell and cell-matrix contacts. Nat Cell Biol 10(5): 527-537.

Seidman JD, Horkayne-Szakaly I, Haiba M, Boice CR, Kurman RJ, Ronnett BM (2004) The histologic type and stage distribution of ovarian carcinomas of surface epithelial origin. Int J Gynecol Pathol 23(1): 41-44.

Venneri MA, Palma MD, Ponzoni M, Pucci F, Scielzo C, Zonari E, Mazzieri R, Doglioni C, Naldini L (2007) Identification of proangiogenic TIE2expressing monocytes (TEMs) in human peripheral blood and cancer. Blood 109(12): 5276-5285.

Wang X, Dai Z, Wu X, Wang K (2016) Distinct RNA transcriptome patterns are potentially associated with angiogenesis in Tie2-expressing monocytes. Gene 580(1): 1-7.

Wang X, Deavers M, Patenia R, Bassett Jr. RL, Mueller P, Ma Q, Wang E, Freedman RS (2006) Monocyte/macrophage and T-cell infiltrates in peritoneum of patients with ovarian cancer or benign pelvic disease. $J$ Transl Med 4: 30.

Wang X, Zhao X, Wang K, Wu L, Duan T (2013) Interaction of monocytes/ macrophages with ovarian cancer cells promotes angiogenesis in vitro. Cancer Sci 104(4): 516-523.

Wolk A, Mantzoros CS, Andersson SO, Bergstrom R, Signorello LB, Lagiou P, Adami HO, Trichopoulos D (1998) Insulin-like growth factor 1 and prostate cancer risk: a population-based, case-control study. J Natl Cancer Inst 90(12): 911-915.

(c) (1)(2) This work is licensed under the Creative Commons (1) Attribution-Non-Commercial-Share Alike 4.0 International License. To view a copy of this license, visit http:// creativecommons.org/licenses/by-nc-sa/4.0/

(C) The Author(s) named above 2017

Supplementary Information accompanies this paper on British Journal of Cancer website (http://www.nature.com/bjc) 